\title{
Ist die Geschlechterrolleneinstellung entscheidend? Die Wirkung länderspezifischer Geschlechterkulturen auf die Erwerbsarbeitszeiten von Frauen
}

\author{
Angelika Kümmerling • Dominik Postels
}

Online publiziert: 24. August 2020

(C) Der/die Autor(en) 2020

Zusammenfassung In der letzten Dekade wurde (nicht nur) in Deutschland eine Reihe von Maßnahmen zur Erhöhung der weiblichen Erwerbsbeteiligung eingeführt (Ausbau der Kinderbetreuungseinrichtungen, Elterngeld etc.). Während sich die Erwerbsquote der Frauen deutlich erhöht hat, fallen die Effekte auf das Arbeitsvolumen deutlich schwächer aus. Ziel des Beitrags ist es, diejenigen Faktoren zu identifizieren, die zu höheren Arbeitszeiten von Frauen beitragen. Hierfür vergleichen wir auf Grundlage des European Social Surveys die Arbeitszeiten von Frauen in der EU-27 und entwickeln ein Mehrebenenmodell, das uns ermöglicht, Einflüsse von Makro- und Mikroebene auf die Arbeitszeiten gleichzeitig zu beobachten. Auf der Mikroebene kontrollieren wir die individuelle Qualifikation, die Lebensphase und verschiedene Arbeitsplatzcharakteristika, auf der Makroebene soziostrukturelle und familienpolitische Unterschiede in den EU-Ländern. Aufbauend auf der Theorie der Geschlechterkulturen von Pfau-Effinger modellieren wir zusätzlich die Geschlechterkultur als mit Makrostrukturen interagierende Variable. Unsere Ergebnisse zeigen, dass die Wirkung familienfreundlicher Maßnahmen, wie die Verfügbarkeit von Kinderbetreuungseinrichtungen oder flexibler Arbeitszeitmodelle, durch länderspezifische Geschlechterrollenvorstellungen beeinflusst werden. Wir schließen daraus, dass politische und betriebliche Maßnahmen zur besseren Einbindung von Frauen in den Arbeitsmarkt und insbesondere zur Erhöhung ihrer Arbeitszeit nur dann ih-

Online-Anhang: www.kzfss.uni-koeln.de/sites/kzfss/pdf/Kuemmerling_Postels.pdf.

\footnotetext{
A. Kümmerling $(\square)$

Institut Arbeit und Qualifikation, Universität Duisburg-Essen

Forsthausweg 2, 47057 Duisburg, Deutschland

E-Mail: Angelika.kuemmerling@uni-due.de

D. Postels

Amt für Statistik, Stadtforschung und Wahlen, Stadt Essen

Porscheplatz, 45121 Essen, Deutschland

E-Mail: Dominik.postels@amt12.essen.de
} 
re volle Wirksamkeit entfalten können, wenn diese durch die in einer Gesellschaft vorherrschenden Rollen- und Familienleitbilder gestützt werden.

Schlüsselwörter Erwerbsbeteiligung von Frauen · Erwerbsarbeitszeiten von Frauen · Mehrebenenanalyse · Internationaler Vergleich · European Social Survey

\title{
Are Gender Roles Decisive? The Impact of Country-Specific Gender Cultures on Women's Working Time
}

\begin{abstract}
In the last decade in Germany as well as other countries, measures have been taken to increase women's participation in the labour market (such as wellpaid parental leave and an increase in childcare institutions). Although the female employment rate has been substantially increasing in Germany, women's working hours have been fairly stable. Against this background, the objective of this article is to identify those parameters that affect women's working time. On the basis of the European Social Survey, we compared the actual working hours of women in the EU 27 and developed a multilevel model that enabled us to observe potential determinants on both the macro and the micro levels at the same time. On the micro level we controlled for education, household situation, and workplace characteristics, and on the macro level for sociostructural and family policy differences. Building on Pfau-Effinger's theory of gender cultures, we additionally modelled a variable "gender culture" and had it interact with macro structural variables. Our results show that the impact of family-friendly measures, such as the use of childcare institutions and flexible working hours, is indeed influenced by a country's gender culture. We conclude from this that political and firm-level measures to better integrate women into the labour market and, in particular, to increase the working time of women can be fully effective only if they are supported by the roles and family models prevailing in a society.
\end{abstract}

Keywords Women's labour market participation - Women's working time · Multilevel analysis · International comparison · European Social Survey

\section{Einleitung}

In den letzten Jahren hat sich die Erwerbsbeteiligung von Frauen in der EU stark erhöht, ein Trend, der noch nicht abgeschlossen scheint, auch wenn die Krise der Jahre 2008/09 diese Entwicklung etwas gebremst hat (OECD 2016). Insbesondere in Deutschland ist die Frauenerwerbstätigkeit stark angewachsen und liegt mittlerweile deutlich über dem EU-Durchschnitt (Eurostat 2019). Gründe für die steigende Erwerbstätigkeit werden in dem steigenden Qualifikationsniveau von Frauen und dem sektoralen Wandel gesehen (Brenke 2015). In Deutschland haben auch die in den letzten Jahren neu eingeführten oder ausgebauten familienpolitischen Instrumente einen positiven Einfluss, da sie einen schnellen Wiedereinstieg in das Erwerbsleben von Müttern nach der Geburt eines Kindes fördern (Kluve und Schmitz 2014; Huebener et al. 2016). Zu nennen sind hier vor allem das zum 01.01.2007 ein- 
geführte Elterngeld, eine Entgeltersatzleistung, die bis zu 14 Monate gezahlt wird, und die stärkere Verbreitung von Kinderbetreuungseinrichtungen im Zusammenhang mit dem seit dem 01.08.2013 geltenden Rechtsanspruch auf einen Kindergartenplatz auch für 1- bis 3-Jährige.

Der alleinige Blick auf die Beschäftigungsquote gibt aber keine Auskunft darüber, ob die Zunahme der Frauenerwerbstätigkeit auch mit einer Zunahme des von Frauen eingebrachten Arbeitsvolumens einhergeht. Die Betrachtung der durchschnittlich geleisteten wöchentlichen Erwerbsarbeitszeiten offenbart, dass die Erfolge hier deutlich geringer ausfallen und Frauen in einer Reihe von Ländern, darunter die Niederlande, Deutschland und Österreich, zu einem hohen Anteil über Teilzeitarbeit in den Arbeitsmarkt integriert sind, deren ambivalente Auswirkungen (Vereinbarkeit auf Kosten beruflicher Entwicklungsmöglichkeiten und eigener finanzieller Absicherung) mittlerweile gut beschrieben sind (BMFSFJ 2011; Hauser 2008; Klenner und Schmidt 2011). Die Faktoren zu kennen, die dazu beitragen, dass Frauen hauptsächlich über eine geringe Stundenanzahl in den Arbeitsmarkt integriert sind, ist aus zweierlei Gründen von hohem Interesse. Erstens kann eine Erhöhung der durchschnittlichen von Frauen geleisteten Erwerbsarbeitszeit dazu beitragen, den in manchen Branchen bereits akuten, in anderen drohenden Fachkräftemangel zu entschärfen. Zweitens erhöhen längere, zumindest vollzeitnahe Arbeitszeiten die finanzielle Unabhängigkeit und mindern langfristig das Risiko der Altersarmut.

Vor diesem Hintergrund ist das Ziel des folgenden Beitrags, diejenigen Faktoren zu identifizieren, die zu einem höheren Umfang der Erwerbsarbeitszeit von Frauen beitragen. Hierfür vergleichen wir die Erwerbsarbeitszeiten von Frauen in der EU27 und entwickeln ein Mehrebenendesign, das uns erlaubt, Einflüsse von Makround Mikroebene auf die Erwerbsarbeitszeiten gleichzeitig zu beobachten. Auf der Makroebene untersuchen wir die Effekte soziostruktureller Länderunterschiede, den Einfluss familienpolitischer Instrumente und die Verbreitung vereinbarkeitsförderlicher Arbeitszeitorganisation in Betrieben. Auf der Mikroebene kontrollieren wir u. a. die Einflüsse der individuellen Qualifikation, der Haushaltsform und Arbeitsplatzcharakteristika. Der hier vertretene theoretische Rahmen baut auf den Arbeiten von Pfau-Effinger (1996) auf und folgt der These, dass das Ausmaß und der Umfang der weiblichen Erwerbsbeteiligung zu einem nicht unbedeutenden Anteil eine Funktion der in einem Land herrschenden Geschlechterkultur sind. Konkret bedeutet das, dass das Ausmaß, in dem soziostrukturelle Rahmenbedingungen und betriebliche Charakteristika sich auf die Dauer der Erwerbsarbeitszeiten von Frauen auswirken, von länderspezifischen Geschlechterrolleneinstellungen beeinflusst wird. Um diese These zu überprüfen, modellieren wir, in Abgrenzung zu anderen Autoren, die soziostrukturelle und kulturelle Einflüsse auf die Arbeitszeiten von Frauen untersuchen (z. B. Boeckmann et al. 2014; Hipp und Leuze 2015), Geschlechterkultur nicht nur als zusätzlich erklärende, sondern als mit Makrostrukturen interagierende Variable. Dieses Vorgehen ermöglicht uns letztlich Rückschlüsse darauf, warum familienpolitische Maßnahmen nicht immer oder nur teilweise zum Erfolg führen (Pfau-Effinger 2012).

Abschnitt 2 des Beitrags stellt aktuelle Befunde und Erklärungsansätze zu länderspezifischen Unterschieden in der Dauer der Erwerbsarbeitszeiten von Frauen dar sowie die Entwicklung unserer Hypothesen. Abschnitt 3 gibt einen Überblick über 
den Stand der Frauenerwerbstätigkeit in der EU, mit einem speziellen Fokus auf ausgewählte Länder. In Abschn. 4 beschreiben wir das von uns entwickelte Analysemodell und das methodische Vorgehen sowie die erhaltenen Ergebnisse. Der Beitrag endet mit der Diskussion der Ergebnisse (Abschn. 5) und ihrer Einbettung in die aktuelle Forschung (Abschn. 6).

\section{Theoretische und empirische Ansätze zur Erklärung weiblicher Erwerbsarbeitszeitausprägungen}

Die Untersuchung der Ausprägungen und Bedingungen weiblicher Erwerbstätigkeit nimmt seit Mitte der 1980er-Jahre einen breiten Raum in der sozialwissenschaftlichen Forschung ein. Insgesamt lassen sich gängige Erklärungsansätze für geschlechtsspezifische Länderdifferenzen in der Erwerbsbeteiligung selbst und des Erwerbsarbeitsumfangs danach unterscheiden, ob sie die Ursachen eher auf der Makro- oder Mikroebene verorten. Dabei konzentrieren sich die Arbeiten jedoch vielfach auf die theoretische Bestimmung potenzieller Einflussfaktoren, wobei ein möglicher Zusammenhang zwischen Theorie und Empirie häufig rein deskriptiv oder korrelativ dargestellt wird (z. B. Dingeldey 1999, 2000; Höllinger 1991; PfauEffinger 1996). In jüngerer Zeit gibt es jedoch vermehrt Bestrebungen, die verschiedenen Einflussfaktoren multivariat oder in einem Mehrebenenmodell, in dem Mikro- und Makrovariablen kombiniert analysiert werden können, zu untersuchen (z. B. Boeckmann et al. 2014; Campa et al. 2011; Hipp und Leuze 2015; Pettit und Hook 2005; Steiber und Haas 2009). Diesen Versuchen ist jedoch gemeinsam, dass sie sich häufig entweder nur auf eine reduzierte Grundgesamtheit oder bestimmte homogene Gruppen von Frauen beziehen (z. B. Frauen mit Kindern oder Frauen in Paarbeziehungen) oder einen „Within-country“-Ansatz (vgl. Gornick et al. 1996) vertreten.

\subsection{Erklärungsansätze auf der Mikroebene}

Auf der Mikroebene werden als Ursachen für unterschiedliche Ausprägungen der Arbeitsmarktbeteiligung von Frauen, insbesondere Müttern, vor allem persönliche Motive oder Präferenzen (z. B. Karriereorientierung vs. Familienorientierung, Leistungsmotiv) sowie Geschlechterrolleneinstellungen untersucht. Dabei zeigen die Ergebnisse allgemein, dass eine hohe Leistungsmotivation mit einer höheren Karriereorientierung und stärkerer Erwerbsbeteiligung einhergeht (Alfermann 1996). Traditionelle und nicht-egalitäre Geschlechterrolleneinstellungen korrelieren ceteris paribus negativ mit der weiblichen Arbeitsmarktbeteiligung (Fortin 2005). Nordenmark (2004) weist in einer multinationalen Studie positive Zusammenhänge zwischen Erwerbstätigkeit, Dauer der Erwerbsarbeitszeit und einer egalitären Geschlechterrolleneinstellung nach. Zudem kann er Unterschiede im Ausmaß dieses Zusammenhangs in Abhängigkeit des Wohlfahrtsstaatenregimes feststellen. In einer US-amerikanischen Längsschnittstudie zeigen Corrigal und Konrad (2007), dass sich eine egalitäre Geschlechterrolleneinstellung positiv auf die Dauer der Erwerbsarbeitszeit von Frauen zu einem späteren Zeitpunkt auswirkt. Stickney und Konrad (2007) können, 
ebenfalls in einer multinationalen Studie, feststellen, dass sich egalitäre Geschlechterrolleneinstellungen positiv auf das Einkommen von Frauen auswirken und dieser Effekt umso stärker ausfällt, je höher die Anzahl geleisteter Stunden ist. Steiber und Haas (2009) stellen zudem fest, dass der Zusammenhang zwischen Einstellung und Erwerbstätigkeit über die Länder hinweg variiert, also in einigen Ländern (hier: liberale Wohlfahrtsstaaten) stärker als in anderen ausfällt. Steiber und Haas erklären dieses Phänomen mit dem Hinweis auf die Theorie der kognitiven Dissonanz, nach der Individuen bestrebt sind, Inkonsistenzen zwischen Einstellungen und Verhalten aufzuheben, insbesondere, wenn es keine externalen Gründe oder Rechtfertigungen für das gezeigte Verhalten gibt (Festinger und Carlsmith 1959). In Ländern, in denen die Erwerbstätigkeit von Müttern nicht durch staatliche Anreize gefördert wird, die als externale Rechtfertigungsgründe für das gezeigte Verhalten dienen könnten, sind erwerbstätige Mütter demnach stärker bestrebt, ihre Einstellungen in Einklang mit ihrem Verhalten zu bringen.

Neuere Studien lassen aber auch eine andere Deutung zu. Demnach handelt es sich bei Geschlechterrolleneinstellungen nicht um ein eindimensionales Konstrukt, das sich auf einer zweipoligen Skala mit den Endpunkten traditionell und egalitär operationalisieren lässt, sondern Geschlechterrolleneinstellungen weisen einen multidimensionalen Charakter auf (Grunow et al. 2018; Knight und Brinton 2017). So identifizieren Yu und Lee (2013) zum Beispiel die Dimensionen ,Einstellung zur Berufstätigkeit von Müttern“ und „Einstellungen zur Gleichverteilung von Haushaltsaufgaben“ und stellen auf Basis von Mehrebenenanalysen fest, dass ,,residents in societies with fewer impediments for women express higher levels of approval of employed mothers, but lower levels for gender equality at home" (Yu und Lee 2013, S. 615). Für unsere Studie ist wichtig, dass diese Untersuchungen zeigen, dass (a) Länder sich hinsichtlich der Ausprägungen des Vorkommens der Dimensionen unterscheiden und (b) innerhalb eines Landes zu einem gegebenen Zeitpunkt unterschiedliche und auch konfligierende Geschlechterrollenvorstellungen vorhanden sein können. Dies wiederum kann dazu führen, dass der Zusammenhang zwischen Einstellung zur Erwerbstätigkeit von Müttern und der tatsächlichen Müttererwerbstätigkeit im Ländervergleich uneinheitlich ausfällt.

Weitere Studien zum Umfang der Erwerbsarbeit von Frauen rekurrieren auf soziodemografische Faktoren und hier insbesondere auf den Haushaltskontext, d.h. auf die Effekte, die von Partnerschaft, Ehe und Kindern auf die Erwerbsbeteiligung und Erwerbsarbeitszeit ausgehen (Anxo et al. 2013; Geyer und Steiner 2007; Grunow et al. 2011; Kümmerling et al. 2008). Der ökonomischen Haushaltstheorie (Becker 1985) zufolge kommt es im Laufe von Paarbeziehungen zu Spezialisierungen als Resultat eines innerpartnerschaftlichen Aushandlungsprozesses, dessen Basis gegenwärtige und zukünftige Verdienstmöglichkeiten darstellen. Der Partner mit dem größeren Verdienstpotenzial (aufgrund von typischen Alters-, Bildungsund Berufswahlunterschieden meist der Mann) spezialisiert sich auf die Erwerbsarbeit, der andere Partner (meist die Frau) auf Haushalt und Sorgearbeit. Entsprechend konnten Anxo et al. (2013) in einem EU-27-Vergleich zeigen, dass die Wahrscheinlichkeit, kurze Teilzeit (bis zu 20h) zu arbeiten, für Frauen in Paarbeziehungen mit Kindern im Vergleich zu Frauen in Paarbeziehungen ohne Kinder signifikant erhöht war. Das Ausmaß des Effektes des Haushaltskontextes variierte allerdings 
über Ländercluster hinweg, sodass anzunehmen ist, dass die Art und Weise, wie Paare oder Individuen ihre Erwerbsarbeitszeitentscheidung treffen, auch von Länderstrukturen beeinflusst wird. Schulze-Buschoff et al. (1998) fanden in einer Strukturgleichungsanalyse, die sie in 11 europäischen Ländern durchführten, in 9 Ländern einen positiven Zusammenhang zwischen sozioökonomischem Status und Vollzeiterwerbstätigkeit. Weiterhin gelten die Qualifikation und das Lebensalter von Frauen als signifikante Einflussfaktoren. Während Analysen zeigen, dass die Dauer der Erwerbsarbeitszeit mit der Qualifikation steigt, ist der Einfluss des Lebensalters jedoch nicht linear (Anxo et al. 2013; Boll et al. 2011; Uunk et al. 2005). Zudem ist die Dauer der Erwerbsarbeitszeit nicht unabhängig von betrieblichen Charakteristika. Anxo et al. (2013) können zeigen, dass die durchschnittliche Länge der Erwerbsarbeitszeit negativ mit der Betriebsgröße zusammenhängt und im Mittel kürzer in Dienstleistungsunternehmen als in Betrieben des Verarbeitenden Gewerbes ausfällt.

Darüber hinaus gibt es Hinweise, dass auch eine familienfreundliche Arbeitszeitorganisation dazu beiträgt, dass Frauen längere Erwerbsarbeitszeiten aufweisen. Es ist derzeit noch nicht hinlänglich geklärt, welche betrieblichen Instrumente tatsächlich in der Lage sind, die Work-Life-Balance von Müttern so zu unterstützen, dass sie systematisch zu einer Erhöhung ihrer wöchentlichen Erwerbsarbeitszeit beitragen. Jüngere Studien weisen jedoch darauf hin (Lott 2019), dass Mütter mit einem gewissen Grad an Autonomie hinsichtlich der Entscheidung, zu welchen Zeiten und an welchem Ort gearbeitet werden kann, längere Erwerbsarbeitszeiten haben als Mütter mit festgelegter Erwerbsarbeitszeit und Arbeitsort.

\subsection{Erklärungsansätze auf der Makroebene}

Ein weiterer Forschungsstrang versucht Unterschiede in der Erwerbsbeteiligung von Frauen durch den Verweis auf institutionelle Unterschiede in den Ländern zu erklären. Hier wird vor allem auf die Ausgestaltung des Wohlfahrtsstaates (EspingAndersen 1990) oder speziell auf die Ausgestaltung familienpolitischer Leistungen rekurriert (vgl. Pfau-Effinger 2000). Dieser Ansatz wurde in der Folge um eine Geschlechterdimension erweitert (z.B. Lewis und Ostner 1994; Sainsbury 1996) und Länder danach unterschieden, ob sie einem starken, moderaten oder schwachen Ernährermodell folgen. Die Ausprägungen des länderspezifischen Ernährermodells können dabei quer zu den Wohlfahrstaaten liegen. Eine wesentliche Annahme ist, dass Sozialpolitik in der Lage ist, „Geschlechterungleichheit zu verringern oder zu verfestigen" (Kulawik 2005, S. 7).

Vor diesem Hintergrund hat sich mittlerweile eine Reihe von Studien mit den Auswirkungen familienpolitischer Leistungen auf die Erwerbstätigkeit und auf den Umfang der Erwerbsarbeitszeiten von Frauen beschäftigt. Die Untersuchungen konzentrieren sich zumeist auf potenzielle Effekte von steuerlichen Systemen oder dem Vorhandensein von (öffentlich finanzierten) Kinderbetreuungseinrichtungen. Bezüglich der Verbreitung von öffentlichen Kinderbetreuungseinrichtungen, insbesondere für Kinder unter 3 Jahren, stellen Uunk et al. (2005) in einer Längsschnittstudie in 13 EU-Ländern fest, dass ihre stärkere Verbreitung mit einem geringeren Rückgang des Umfangs der Erwerbsarbeit nach der Geburt des ersten Kindes einhergeht (ähnlich auch Attanasio et al. 2008; Boeckmann et al. 2014; Hipp und Leuze 2015; 
Lefebvre und Merrigan 2008). Havnes und Mogstad (2009) können für Norwegen dagegen keinen kausalen Effekt zwischen der Bereitstellung universeller Kinderbetreuung und mütterlicher Erwerbstätigkeit feststellen. Gleichfalls zeigt Jaumotte (2003) in ihrer Zusammenstellung der Wirkung unterschiedlicher familienpolitischer und sozialstaatlicher Instrumente auf die Erwerbstätigkeit von Frauen, dass zwar ein Zusammenhang zwischen staatlichen Subventionen von Kinderbetreuungseinrichtungen und Müttererwerbstätigkeit existiert, dieser aber keinesfalls linear ist: „The empirical evidence shows that childcare subsidies do increase female labour supply, though in some instances their effectiveness is reduced as, for example, women substitute formal for informal childcare" (Jaumotte 2003, S. 9). Pettit und Hook (2005) kommen in ihrer Analyse von 19 Ländern zu dem Ergebnis, dass öffentlich finanzierte Kinderbetreuung positiv mit der Erwerbstätigkeit von Müttern zusammenhängt, der Zusammenhang zwischen Elternzeit oder Erziehungsurlaub und Erwerbstätigkeit jedoch den Verlauf einer umgedrehten U-Kurve nimmt. Die Dauer der Elternzeit ist also entscheidend dafür, ob sie positive oder negative Effekte auf die Beschäftigung ausübt. Boeckmann et al. (2014) stellen in einer Analyse von 18 OECD-Ländern fest, dass eine vergleichsweise kurze, aber gut bezahlte Elternzeit mit einer stärkeren Erwerbsneigung und längeren Erwerbsarbeitszeiten von Müttern verbunden ist. Eine jüngere, experimentelle Studie von Hipp (2018) über die Auswirkungen der Länge der genommenen Elternzeit auf Karrierechancen von Müttern und Vätern in Deutschland zeigt dagegen, dass Mütter, die nach der Geburt eines Kindes nur einen sehr kurzen Zeitraum pausieren, stärkere Schwierigkeiten bei der Suche nach einem neuen Job zu erwarten haben, als Mütter, die die volle Elternzeit von 12 Monaten genommen haben.

Das Steuerungspotenzial von Steuersystemen hinsichtlich der Aufteilung der Erwerbsarbeit in Paaren hat Dingeldey $(1999,2000)$ in einer internationalen Studie untersucht. Verkürzt dargestellt begünstigt die individuelle Besteuerung von Paaren die Erwerbstätigkeit und ihren Umfang, während die gemeinsame Besteuerung Einverdienerhaushalte oder eine ungleiche Aufteilung der Erwerbstätigkeit innerhalb der Paare bevorteilt (Jaumotte 2003). Auch Hipp und Leuze (2015) können zeigen, dass Unterschiede in der Erwerbsarbeitszeit zwischen Paaren in denjenigen Ländern höher ausfallen, in denen Paare gemeinsam besteuert werden.

Während Unterschiede in der Arbeitsmarktteilhabe von Frauen in Abhängigkeit familienpolitischer sowie soziostruktureller Gegebenheiten in der sozialwissenschaftlichen Literatur mittlerweile gut belegt sind und zunehmend Informationen darüber verfügbar sind, welche Instrumente die Erwerbstätigkeit von Frauen und Müttern fördern (u.a. Boeckmann et al. 2014; Hipp und Leuze 2015; Ziefle und Gangl 2014), bleibt jedoch bislang unklar, wie genau Wohlfahrts- oder Sozialstrukturen ein bestimmtes Verhalten von Frauen und Männern beeinflussen und transportieren und warum manche Maßnahmen nicht den gewünschten Effekt erzielen.

In ihrer Theorie über Geschlechterkulturen kritisiert Pfau-Effinger die Sichtweise, dass die individuelle Ausgestaltung der Erwerbstätigkeit ,im wesentlichen nur ein Reflex wohlfahrtsstaatlicher Politiken“ sei (Pfau-Effinger 2000, S. 20), da es Handeln rein auf Zweckrationalität und ökonomische Kalküle verkürze: Sozialstaatliche Instrumente, wie z.B. die gemeinsame oder getrennte Besteuerung von Ehepaaren, die öffentliche Förderung von (Kinder-)Betreuungseinrichtungen oder die Verbrei- 


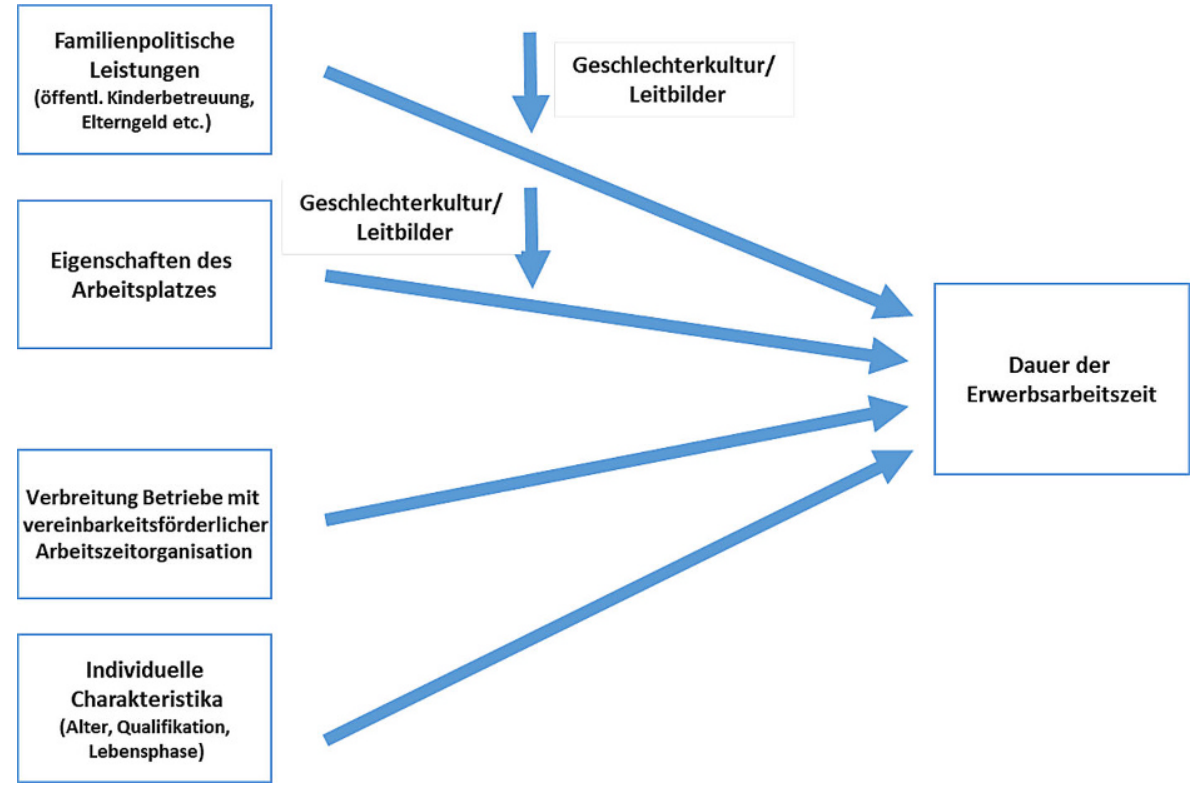

Abb. 1 Determinanten der weiblichen Erwerbsarbeitszeit

tung von Ganztagsschulen, können ihrer Meinung nach nur Anreize für ein Verhalten setzen. Inwieweit dieses Verhalten dann von den Mitgliedern einer Gesellschaft auch gezeigt wird, sei wiederum abhängig von individuellen Dispositionen (z. B. familiale vs. Erwerbsorientierung, Bildungsgrad, persönliche Arbeitsmarktchancen), der Haushaltssituation (z.B. Notwendigkeit der Erwerbstätigkeit unabhängig von der persönlichen Neigung) und Arbeitsmarktangeboten (Teilzeit- vs. Vollzeitarbeitsplätze). „[T]he actual outcomes of family policies can differ from their aims and the incentives they set. There is much evidence that women in some countries or regions behave differently to what one would expect" (Pfau-Effinger 2012, S. 532). Vor diesem Hintergrund formuliert Pfau-Effinger die These, dass ,sich das Erwerbshandeln von Frauen zu einem erheblichen Anteil auf kulturelle Leitbilder darüber bezieht, welches die wünschenswerte, ,richtige" Arbeitsteilung zwischen den Geschlechtern ist, und daß solche Leitbilder im Ländervergleich variieren“ (1996, S. 464). Dabei bilden diese Leitbilder kein isoliertes emergentes Konstrukt, sondern es ist davon auszugehen, dass die in einer Gesellschaft dominierenden Geschlechterleitbilder in das jeweilige Wohlfahrtsstaatsarrangement eines Landes integriert sind oder es zumindest über einen längeren Zeitraum hinweg waren. Einerseits können sich wohlfahrtsstaatliche Arrangements schneller als die Leitbilder ändern (ein Beispiel hierfür findet sich in der Transformation der DDR zu einem Teilgebiet der Bundesrepublik), andererseits können sich gesellschaftliche Rollenverständnisse schneller wandeln, als es die gesellschaftlichen Strukturen tun. Leitbilder stellen also eine Dimension von sozialen Institutionen dar, müssen ihnen aber nicht entsprechen. Desgleichen ist davon auszugehen, dass die strukturellen Rahmenbedingungen eines Wohlfahrtsstaates in Wechselwirkung mit den herrschenden Rollenleitbildern stehen und nur 
dann ihre volle Wirkung entfalten können, wenn sie im Einklang mit in einer Gesellschaft herrschenden Normen und Leitbildern stehen. Maßnahmen zur Verbesserung der Vereinbarung von Beruf und Familie laufen demnach Gefahr zu verpuffen, wenn sie nicht mit der in einer Gesellschaft herrschenden Ideologie über die ,gute“ oder „ideale“ Mutter übereinstimmten (Macdonald 2009, S. 411). Ein Beispiel hierfür ist die Steuerreform in den Niederlanden, die 2001 mit dem Ziel eingeführt wurde, Arbeit für Frauen mit einem gutverdienenden Partner attraktiver zu machen, jedoch darin resultierte, dass ,women even slightly reduced their working hours in response to receiving a higher after-tax hourly wage“" (Booth und van Ours 2010, S. 4).

Auf diesen Annahmen aufbauend entwickeln wir ein Mehrebenendesign, das uns erlaubt, Einflüsse von Makro- und Mikroebene gleichzeitig zu beobachten. Im Einklang mit der Theorie Pfau-Effingers (1996, 2012) behandeln wir die Geschlechterkultur nicht allein als zusätzlich erklärende Variable, sondern lassen sie mit Variablen, die Makrostrukturen abbilden, interagieren (sog. Cross-Level-Effekte). Das heißt, wir operationalisieren für unsere Zwecke Geschlechterkulturen als Größe, die den Effekt der unabhängigen Variablen (z. B. familienpolitische Instrumente, vereinbarkeitsförderliche Arbeitszeitorganisation) auf die abhängige Variable (Dauer der Erwerbsarbeitszeit) beeinflusst (vgl. Abb. 1).

Vor diesem Hintergrund lauten unsere Hypothesen:

H1 Der Umfang der wöchentlichen Erwerbsarbeitszeit von Frauen wird durch den Haushaltskontext beeinflusst. Konkret erwarten wir, dass Partnerschaft und Ehe sowie betreuungspflichtige Kinder im Haushalt in einem negativen Zusammenhang mit der Dauer der wöchentlichen Erwerbsarbeitszeit stehen.

H2 In Ländern, in denen eine progressive Geschlechterkultur herrscht, sind die wöchentlichen Erwerbsarbeitszeiten von Frauen länger.

H3 Soziostrukturelle Rahmenbedingungen und insbesondere familienpolitische Leistungen wirken sich auf die Dauer der Erwerbsarbeitszeiten von Frauen aus. In Übereinstimmung mit der Literatur gehen wir davon aus, dass sich eine hohe Verbreitung öffentlicher Kinderbetreuungseinrichtungen positiv auf den Umfang der Erwerbsarbeitszeiten von Frauen auswirkt (Hypothese 3a). Außerdem erwarten wir einen positiven Effekt von finanziell kompensierter Elternzeit (,well paid leave“) auf den Umfang der Erwerbsarbeitszeiten von Frauen (Hypothese 3b). Bezüglich der Besteuerung von Paaren gehen wir dagegen von einem negativen Zusammenhang zwischen gemeinsamer steuerlicher Veranlagung und dem Umfang der Erwerbsarbeitszeit von Frauen aus (Hypothese 3c).

H4 Neben familienpolitischen Leistungen spielt auch die Verbreitung einer vereinbarkeitsförderlichen Arbeitszeitorganisation in den Betrieben eine Rolle. Instrumente einer familienförderlichen Arbeitszeitorganisation sind z. B. Maßnahmen, die mit mehr Autonomie der Beschäftigten hinsichtlich Start- und Endzeitpunkt der täglichen Erwerbsarbeitszeit verbunden sind. Wir gehen daher davon aus, dass der Umfang der wöchentlichen Erwerbsarbeitszeiten von 
Frauen positiv mit der Verbreitung von Gleitzeitregelungen (Hypothese 4a) und Nutzung von flexiblen Arbeitszeitformen zusammenhängt (Hypothese 4b).

H5 Die von Frauen erzielte wöchentliche Erwerbsarbeitszeitdauer ist sowohl abhängig von der individuellen Haushaltssituation als auch den gegebenen länderspezifischen soziostrukturellen und betrieblichen Rahmenbedingungen. Das Ausmaß, in dem die länderspezifischen Rahmenbedingungen die Dauer der Erwerbsarbeitszeit beeinflussen, wird wiederum von der in einem Land herrschenden Geschlechterkultur determiniert (Cross-Level-Interaktion).

Wie in Abschn. 2 dargestellt, wird in der Literatur zusätzlich zu den in den Hypothesen genannten Einflussgrößen noch eine Reihe weiterer Faktoren genannt, die den Umfang der Erwerbsarbeitszeit von Frauen beeinflussen können und die wir hier als Kontrollvariablen in die Gleichung aufnehmen.

Auf der individuellen Ebene ist dies zunächst das Alter der Befragten, das wir sowohl als Lebensalter als auch quadriert in die Formel einführen, da Analysen zeigen, dass die Auswirkungen des Alters auf die Erwerbsarbeitszeiten nicht linear sind (vgl. Anxo et al. 2013). Zudem kontrollieren wir für die Bildung der Beschäftigten und gehen davon aus, dass höhere Bildung sich positiv auf die Dauer der Erwerbsarbeitszeiten auswirkt. Da es Hinweise dafür gibt, dass Erwerbsentscheidungen von Frauen nicht unabhängig von der finanziellen Situation des Haushalts getroffen werden (Dex und Joshi 1999; Wippermann 2018), nehmen wir auch die subjektive Einschätzung des Haushaltseinkommens als Kontrollvariable auf. Außerdem kontrollieren wir betriebliche Faktoren, wie Betriebsgröße und Branchenzugehörigkeit des Betriebes, da die Dauer der Erwerbsarbeitszeit mit diesen Faktoren systematisch variiert (die durchschnittlichen Erwerbsarbeitszeiten sind kürzer in Dienstleistungsunternehmen und länger in größeren Betrieben; Kümmerling et al. 2017; Eurostat 2019).

\section{Erwerbsbeteiligung von Frauen in der EU}

Bevor wir zur allgemeinen Hypothesentestung übergehen, stellen wir in diesem Abschnitt zentrale deskriptive Befunde zur Frauenerwerbstätigkeit und zu Erwerbsarbeitszeiten in der EU dar. Im Jahr 2014 waren EU-weit 63,5\% aller Frauen zwischen 20 und 64 Jahren erwerbstätig - Spitzenreiter mit Werten deutlich über $70 \%$ waren die Nordischen Länder Schweden, Dänemark und Finnland, aber auch Deutschland auf Rang 2. Mit einer Erwerbsquote von deutlich unter $50 \%$ bildete Griechenland das Schlusslicht. Rechnet man jedoch die Erwerbsquoten auf Vollzeitstellen um, in die sogenannten Vollzeitäquivalenzraten ${ }^{1}$ (VZÄR, Tab. 1, Spalte 3), stellt sich die Frauenerwerbsbeteiligung nunmehr sehr unterschiedlich dar.

\footnotetext{
1 Im Unterschied zur Erwerbstätigenquote handelt es sich bei der Vollzeitäquivalenzrate um eine Maßzahl, die nicht nur Information darüber enthält, wie hoch der Anteil derjenigen ist, die einer Erwerbstätigkeit nachgehen, sondern auch den Umfang der Erwerbstätigkeit mit einbezieht. In diesem Sinne definiert Eurostat Vollzeitäquivalente als ,total hours worked on both main and second job divided by the average annual number of hours worked in full-time jobs“ (Europäische Kommission 2012, S. 437), also die gesamten durch die Gruppenmitglieder gearbeiteten Stunden in ihrer Haupt- $\left(x_{1 i}\right)$ und Nebenerwerbstätigkeit $\left(x_{2 i}\right)$,
} 
Tab. 1 Erwerbs- und Teilzeitquoten, Vollzeitäquivalenzraten und durchschnittliche wöchentliche Erwerbsarbeitszeiten von Frauen in der EU (2014). (Quelle: Eurostat 2019)

\begin{tabular}{|c|c|c|c|c|}
\hline Land & $\begin{array}{l}\text { Erwerbsquote (in \%) } \\
\text { (20- bis 64-Jährige) }\end{array}$ & $\begin{array}{l}\text { Teilzeitquote (in \%) } \\
\text { (20- bis 64-Jährige) }\end{array}$ & $\begin{array}{l}\text { VZÄR }{ }^{\mathrm{a}} \text { (in \%) } \\
\text { (15- bis 64- } \\
\text { Jährige) }\end{array}$ & $\begin{array}{l}\text { Wöchentliche } \\
\text { Erwerbsarbeits- } \\
\text { zeiten (in h) }\end{array}$ \\
\hline SE & 77,6 & 35,8 & 60,6 & 34,3 \\
\hline $\mathrm{DE}$ & 73,1 & 46,7 & 50,3 & 30,2 \\
\hline DK & 72,2 & 31,2 & 54,1 & 30,7 \\
\hline FI & 72,1 & 17,5 & 57,1 & 34,6 \\
\hline $\mathrm{EE}$ & 70,6 & 10,9 & 62,1 & 37,9 \\
\hline LT & 70,6 & 10,6 & 57,9 & 37,4 \\
\hline UK & 70,6 & 40,0 & 47,3 & 32,1 \\
\hline AT & 70,1 & 47,2 & 48,5 & 31,5 \\
\hline NL & 69,8 & 75,2 & 40,3 & 24,1 \\
\hline LV & 68,5 & 8,8 & 59,6 & 38,5 \\
\hline FR & 66,2 & 31,1 & 49,4 & 33,7 \\
\hline LU & 65,5 & 35,2 & 45,4 & 33,6 \\
\hline $\mathrm{CZ}$ & 64,7 & 9,4 & 53,2 & 38,3 \\
\hline PT & 64,2 & 12,4 & 53,0 & 38,4 \\
\hline CY & 63,9 & 16,5 & 50,5 & 38,3 \\
\hline SI & 63,6 & 13,0 & 52,6 & 38,4 \\
\hline EU-28 & 63,5 & 31,8 & 49,4 & 33,2 \\
\hline $\mathrm{BE}$ & 62,9 & 41,1 & 44,9 & 32,1 \\
\hline BG & 62,0 & 2,7 & 55,3 & 40,2 \\
\hline IE & 61,2 & 33,6 & 41,5 & 31,5 \\
\hline $\mathrm{HU}$ & 60,2 & 8,3 & 52,6 & 38,9 \\
\hline PO & 59,4 & 10,2 & 48,6 & 38,3 \\
\hline SK & 58,6 & 6,7 & 49,3 & 38,8 \\
\hline RO & 57,3 & 9,2 & 49,3 & 40,5 \\
\hline ES & 54,8 & 25,3 & 40,7 & 33,7 \\
\hline HR & 54,2 & 6,7 & 46,3 & 39,4 \\
\hline MT & 52,0 & 28,0 & 39,3 & 34,1 \\
\hline IT & 50,3 & 32,1 & 36,4 & 31,8 \\
\hline GR & 44,3 & 12,9 & 36,0 & 37,1 \\
\hline
\end{tabular}

$V Z \ddot{A} R$ Vollzeitäquivalenzraten, $A T$ Österreich, $B E$ Belgien, $B G$ Bulgarien, $C Y$ Zypern, $C Z$ Tschechien, $D E$ Deutschland, DK Dänemark, EE Estland, ES Spanien, FI Finnland, FR Frankreich, GR Griechenland, $H R$ Kroatien, $H U$ Ungarn, IE Irland, IT Italien, $L T$ Litauen, $L U$ Luxemburg, $L V$ Lettland, $M T$ Malta, $N L$ Niederlande, $P L$ Polen, $P T$ Portugal, $R O$ Rumänien, SE Schweden, SI Slowenien, SK Slowakei, $U K$ Großbritannien

aeigene Berechnungen auf Basis des Labour Force Survey (LFS)

Zunächst ist festzustellen, dass die VZÄR deutlich von den Erwerbsquoten abweichen, am höchsten sind die Differenzen in Malta, den Niederlanden, Großbritannien

geteilt durch die durchschnittlich in Vollzeitbeschäftigungen gearbeiteten Stunden $\left(\bar{x}_{3}\right)$. Analog zur besagten Erwerbstätigenquote lässt sich aus dieser Maßzahl die Vollzeitäquivalenzrate (VZÄR) der Gruppe berechnen, indem die Vollzeitäquivalente der Gruppe in Relation zur Gruppengröße $(n)$ gesetzt werden (vgl. Europäische Kommission 2012, S. 437; ausführlich Kümmerling und Lazarevic 2016). 
und Deutschland, am geringsten in den osteuropäischen Ländern Bulgarien, Ungarn, Kroatien und Rumänien. Die höchsten VZÄR hat Estland, gefolgt von Schweden und Lettland, während Deutschland bei den VZÄR nur noch leicht über dem EUDurchschnitt liegt. Am Ende der Skala liegen wiederum Italien, Griechenland und Malta.

Die Tabelle zeigt auch, dass die Differenz zwischen Beschäftigungsquote und Vollzeitäquivalenzraten über die Länder hinweg stark variiert - ein Indikator für die unterschiedlichen Lösungen, die Länder haben, um Frauen und Mütter in den Arbeitsmarkt zu integrieren.

Ursächlich für diese Diskrepanz ist die in einigen Ländern sehr hohe Teilzeitquote, die wiederum in hohem Maße auf die steigende Erwerbsbeteiligung von Müttern zurückzuführen ist (z. B. Klenner und Lillemeier 2015). Ein nur langsam ansteigendes Arbeitsstundenvolumen verteilt sich also auf eine wesentlich schneller anwachsende Zahl von Köpfen. Zwar ist die zunehmende Frauenerwerbstätigkeit vor dem Hintergrund des demografischen Wandels, aber auch aus gleichstellungspolitischen Gründen, positiv zu bewerten, allerdings wirft die Zunahme von Teilzeitarbeit auch Fragen auf. Denn neben einem aktuell geringeren Einkommen geht Teilzeitarbeit mit eingeschränkten beruflichen Entwicklungs- und Karrierechancen sowie einem höheren Altersarmutsrisiko durch niedrigere Rentenansprüche einher (Frommert et al. 2013; Koch 2008; Lewicki und Wigger 2013). Klenner und Schmidt (2012) zeigen zudem, dass Teilzeitarbeit und insbesondere Minijobs zu Pfadabhängigkeiten in den Erwerbsverläufen von Frauen führen. Teilzeitarbeit - meist eingegangen, um aktuelle Vereinbarkeitsprobleme zwischen Beruf und Familie zu lösen - scheint eine Sogkraft zu entwickeln, sodass Frauen sehr lange in Teilzeitarbeit verharren (Boll 2014).

3.1 Geschlechtsspezifische Erwerbsarbeitszeiten im internationalen Vergleich und in Abhängigkeit des Haushaltstypus

Die Unterscheidung der Erwerbsarbeitszeit allein nach Voll- und Teilzeit und die Betrachtung ihrer durchschnittlichen Verteilung verschleiert die große Heterogenität, die für die Dauer weiblicher Erwerbsarbeitszeiten in vielen Ländern typisch ist (Anxo et al. 2007; Kümmerling 2018). Denn Teilzeitarbeit ist nicht gleich Teilzeitarbeit. Sie kann, wie in Schweden, vollzeitnah ausgerichtet sein, oder, wie in Deutschland und den Niederlanden, sich eher an einem 50-Prozent-Modell der Normalarbeitszeit orientieren. In Tab. 1 sind die durchschnittlichen normalerweise gearbeiteten Wochenarbeitszeiten $^{2}$ von Frauen und Männern in der EU für das Jahr 2014 dargestellt. Auf den ersten Blick ist auffällig, dass die Erwerbsarbeitszeiten von Frauen in der EU stark variieren. Zudem weisen die Erwerbsarbeitszeiten ein starkes OstWest-Gefälle auf: Die Länder, in denen Frauen besonders kurze durchschnittliche Arbeitszeiten aufweisen (unter dem EU-28-Mittelwert liegend), gehören sämtlich zu den alten EU-12-Staaten. Zwischen den Niederlanden, dem Land mit den kürzes-

\footnotetext{
${ }^{2} \mathrm{Zu}$ methodischen Erwägungen hinsichtlich der Messung von Arbeitszeiten vgl. Schief (2003) sowie Kümmerling und Lazarevic (2016).
} 
ten und Rumänien, dem Land mit den längsten Frauenerwerbsarbeitszeiten, liegen durchschnittlich 16,4 Wochenstunden.

Erwerbsarbeitszeiten und Erwerbsquoten stellen Messungen auf hoher Aggregatsebene dar, die die tatsächliche Arbeitsmarktintegration relevanter Teilgruppen unterschlagen können. Dies wird deutlich, wenn man die Arbeitsmarktintegration von Frauen und Männern in einer Lebensverlaufsperspektive betrachtet. Im Zusammenhang mit der Beschreibung und Analyse der Frauenerwerbstätigkeit ist hier insbesondere die Teilhabe von Müttern und Vätern kleiner Kinder zu nennen. Die folgenden Analysen orientieren sich an dem von Anxo et al. (2007) entwickelten Ansatz der lebensphasenspezifischen Haushaltstypologien, der Querschnittsdaten für Lebensverlaufsbeschreibungen nutzbar macht. Dieser Ansatz beschreibt den Lebensverlauf anhand einer Sequenz von unterschiedlichen Haushaltstypen, die typischerweise von der großen Mehrzahl der Menschen in ihrer Biografie durchlebt werden. Solche Haushaltstypen sind z. B. Singlehaushalte, Paarhaushalte ohne oder mit Kindern unterschiedlichen Alters, ältere Paare, die nicht (mehr) mit Kindern im Haushalt leben, und Paare, die sich vor dem Eintritt in die Rentenphase befinden. Da es sich bei der ausgewerteten Datenbasis um Querschnittsdaten handelt, soll noch einmal darauf hingewiesen werden, dass wir keinen Verlauf darstellen, sondern nur Haushaltstypen unterscheiden. Allerdings zeigt der Zeitvergleich, dass sich die dargestellten lebensphasenspezifischen Muster im Zeitverlauf als erstaunlich stabil erwiesen haben (Kümmerling 2018). Für die folgende Analyse haben wir exemplarisch 4 Länder ausgewählt, die sämtlich eine überdurchschnittlich hohe Frauenerwerbstätigkeit aufweisen (Tab. 1), denen also eine vergleichsweise starke Integration von Frauen in den Arbeitsmarkt gelungen ist: Deutschland, ${ }^{3}$ Schweden, Frankreich und Estland. Wir haben für die Darstellung speziell Länder mit einer überdurchschnittlichen Frauenerwerbstätigenquote ausgewählt, um zu zeigen, dass Unterschiede im Erwerbsarbeitszeitumfang von Frauen in unterschiedlichen Haushaltskonstellationen nicht mit der Höhe der Erwerbstätigenquote in einem Land zusammenhängen.

Die durchschnittlichen Erwerbsarbeitszeiten für deutsche, französische, schwedische und estnische Beschäftigte sind in Abb. 2 für 9 unterschiedliche Haushaltstypen dargestellt und zeigen stark kontrastierende Muster. Zunächst fällt auf, dass mit Ausnahme der 35- bis 44-jährigen Singles in Estland in jeder Lebensphase Männer längere Erwerbsarbeitszeiten aufweisen als Frauen. Zudem wird deutlich, dass der Einfluss der Lebensphase die Erwerbsarbeitszeiten von Frauen stärker beeinflusst als die der Männer - und dass dieser Einfluss über die ausgewählten Länder hinweg unterschiedlich hoch ausfällt, mit Abstand am deutlichsten jedoch in Deutschland. Die Auseinanderdifferenzierung der Erwerbsarbeitszeiten fällt nicht erst in die Lebensphase der Familiengründung, sondern lässt sich bereits in der Haushaltsform

\footnotetext{
3 Dabei verbergen die sich auf Gesamtdeutschland beziehenden Werte, dass es hinsichtlich der Arbeitsmarktintegration von Frauen und Müttern nach wie vor substanzielle Unterschiede zwischen Ost- und Westdeutschland gibt, die insbesondere über die verschiedenen Haushaltsformen stark ausgeprägt sind. Frauen in Ostdeutschland weisen in jeder Haushaltskonstellation durchschnittlich höhere Wochenarbeitszeiten auf als Frauen in Westdeutschland (Kümmerling et al. 2015). Ähnliche regionale Unterschiede im weiblichen Erwerbsverhalten sind auch für Italien festgestellt worden, wobei hier die Trennlinie zwischen Norden und Süden verläuft (Fernández 2012).
} 
Abb. 2 Geschlechtsspezifische Unterschiede in den Erwerbsarbeitszeiten nach Lebensphase in ausgewählten Ländern (2014). a Deutschland, b Frankreich, c Schweden, d Estland

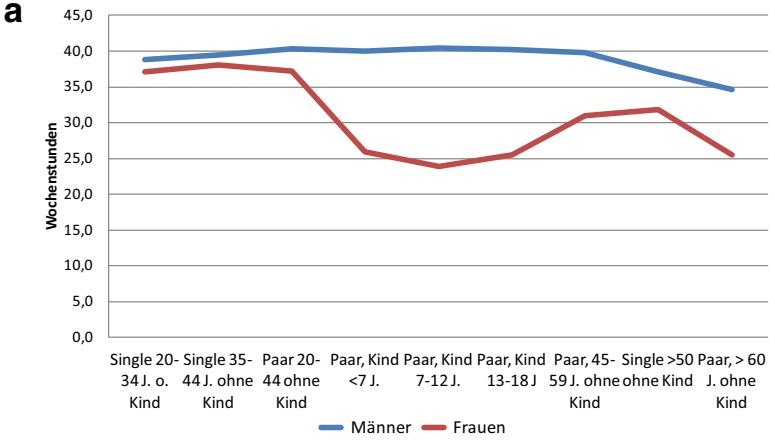

b

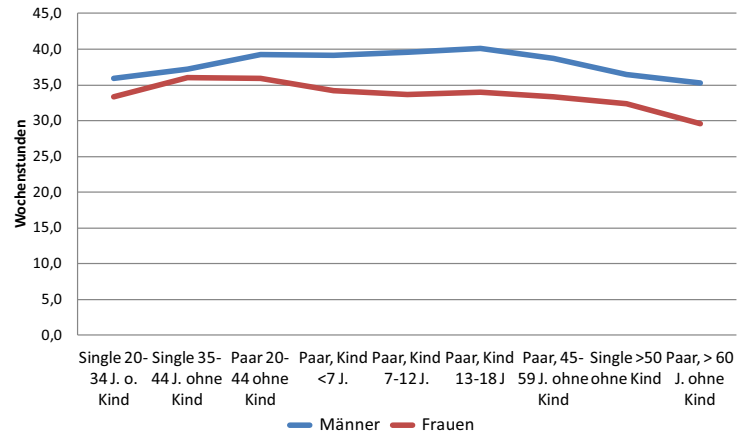

C
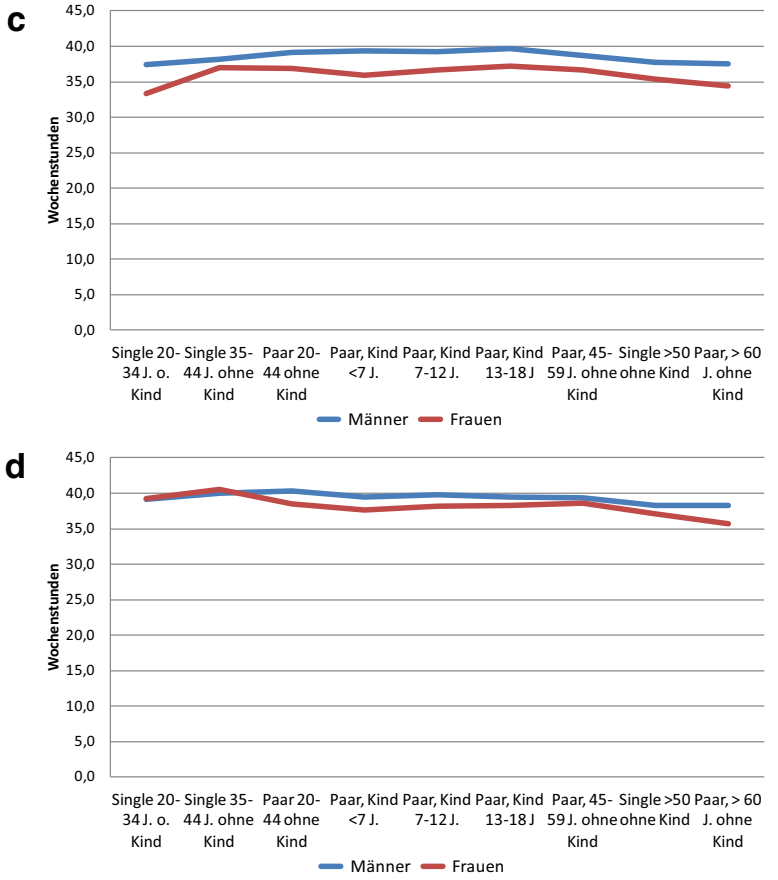
„Paarhaushalt ohne Kinder“ konstatieren - dies ist im Übrigen für alle 4 ausgewählten Länder, allerdings wiederum im unterschiedlichen Ausmaß, festzustellen (vgl. hier für Deutschland die Ergebnisse von Allmendinger und Haarbrücker 2013). Am höchsten fallen die Unterschiede in Frankreich und Deutschland aus, wo Frauen in Paarbeziehungen ohne Kinder im Alter von 20-44 Jahren im Durchschnitt etwas über $3 \mathrm{~h}$ kürzer als Männer arbeiten. Kinder jeden Alters erhöhen die Differenz insbesondere in Deutschland deutlich, sodass Frauen in Haushalten mit Kindern in Deutschland im Durchschnitt zwischen 14,2 und 16,5 h, in Frankreich zwischen 4,9 und 6,2 h, in Schweden zwischen 2,5 und 3,5 h und in Estland nur zwischen 1,1 und $1,8 \mathrm{~h}$ kürzer als Männer arbeiten.

Die Betrachtung des Umfangs der Erwerbsarbeitszeit von abhängig Beschäftigten über die verschiedenen Haushaltstypen hinweg macht deutlich, dass der Familienstand und die Anwesenheit betreuungspflichtiger Kinder im Haushalt sich in sehr unterschiedlichem Ausmaß auf die Erwerbsarbeitszeiten von Frauen auswirken und dass eine hohe Erwerbstätigenquote nicht notwendigerweise mit einer geringen Wochenstundenzahl von Frauen und insbesondere Müttern einhergehen muss. AnschlieBend an diese deskriptiven Analysen stellt sich die Frage, worauf diese deutlichen Unterschiede zurückzuführen sind.

\section{Methode und Daten}

Um den Zusammenhang zwischen wohlfahrtsstaatlichen und familienpolitischen Regelungen, Arbeitsplatzcharakteristika, soziostrukturellen Rahmenbedingungen, individuellen Faktoren und dem Umfang der Erwerbsarbeitszeit von Frauen zu untersuchen, verwenden wir mehrebenenanalytische Verfahren (Raudenbusch und Bryk 2002). Diese erlauben es uns, Individual- und Ländervariablen simultan zu modellieren und den hierarchischen Charakter der Daten zu berücksichtigen. Die Mehrebenenanalyse hat gegenüber der linearen Regression den Vorteil, dass die Signifikanz nicht überschätzt wird, da die zur Berechnung des Standardfehlers herangezogene Stichprobengröße anstatt der Anzahl der Individuen der Anzahl der Länder entspricht (Braun et al. 2010). Geschätzt wird folgendes Modell:

$$
\begin{aligned}
\text { wöchentliche Erwerbsarbeitszeit } \mathrm{i}_{\mathrm{ij}}= & \gamma_{00}+\gamma_{p 0} X_{\mathrm{pij}}+\gamma_{0 q} Z_{\mathrm{qj}}+\gamma_{\mathrm{pq}} Z_{\mathrm{qj}} X_{\mathrm{pij}} \\
& +u_{\mathrm{pj}} X_{\mathrm{pij}}+u_{0 \mathrm{jpq}}+e_{\mathrm{ij}} .
\end{aligned}
$$

Die abhängige Variable ist die Dauer der Erwerbsarbeitszeit von Frauen für Individuen $i$ im Land $j, \gamma_{00}$ ist die durchschnittliche Erwerbsarbeitszeit von Frauen über die Länder hinweg, $\gamma_{p 0} X_{\text {pij }}$ bezeichnet die Koeffizienten sowie die Werte der unabhängigen Variablen $p$ der Individualebene (Partner im Haushalt, Kinder nach Altersgruppen, Alter, Betriebsgröße, Bildung, Sektor und subjektive Einschätzung des Haushaltseinkommens) für die Beobachtungseinheit $i$ im Land $j \cdot \gamma_{0 q} Z_{q j}$ führt die unabhängigen Variablen $q$ der Kontextebene ein, wobei $Z_{j}$ die Werte der unabhängigen Variablen für Land $j$ wiedergibt. Cross-Level-Interaktionen zwischen Individualebene und Kontextfaktoren werden durch $\gamma_{p q} Z_{q j} X_{\mathrm{pij}}$ dargestellt. Mit $u_{p j} X_{\mathrm{pij}}$ werden Geschlechterrolleneinstellungen als Random Slope modelliert, da 
wir annehmen, dass die Einstellungen je nach Länderzugehörigkeit unterschiedlich stark auf die abhängige Variable wirken. $u_{0 j}$ und $e_{i j}$ sind die Fehlerterme der Kontext- bzw. der Individualebene.

In der Analyse kommen verschiedene Datenquellen zum Einsatz. Daten auf der Individualebene stammen aus dem European Social Survey (ESS). Der ESS ist eine repräsentative Querschnittserhebung, die seit 2001 alle 2 Jahre mit unterschiedlichen Themenschwerpunkten in Europa durchgeführt wird. Für das Jahr 2010 stehen 21 Länder zur Verfügung. Daten aus Lettland und Rumänien stammen aus dem Jahr 2008. Insgesamt gehen somit 23 Länder und 9434 Befragte in unsere Analyse ein: Belgien $(n=383)$, Bulgarien $(n=463)$, Dänemark $(n=362)$, Deutschland $(n=630),{ }^{4}$ Estland $(n=426)$, Finnland $(n=383)$, Frankreich $(n=424)$, Griechenland $(n=350)$, Irland $(n=433)$, Lettland $(n=482)$, Litauen $(n=435)$, Niederlande $(n=479)$, Polen $(n=328)$, Portugal $(n=376)$, Rumänien $(n=463)$, Slowakei $(n=403)$, Slowenien $(n=287)$, Schweden $(n=378)$, Spanien $(n=355)$, Tschechien $(n=507)$, Ungarn $(n=329)$, Großbritannien $(n=555)$ und Zypern $(n=203)$. Italien, Malta, Luxemburg und Österreich mussten aufgrund des Fehlens zentraler Variablen aus der Analyse ausgeschlossen werden. In die Analyse gingen nur abhängig beschäftigte Frauen im Alter von 18-65 Jahren ein, die mindestens 1 Stunde pro Woche gearbeitet haben.

Zwar steht im Zentrum der Analyse der Einfluss institutioneller Faktoren auf den Umfang der Erwerbsarbeitszeiten von Frauen, wir kontrollieren jedoch ebenfalls für Faktoren auf Individual- oder Haushaltsebene, um sicherzustellen, dass die länderübergreifende Variation nicht auf Unterschieden zwischen Individuen oder Haushalten beruht. ${ }^{5}$ Auf der Individualebene werden der Beziehungsstatus (= 1 lebt mit Partner zusammen; = 0 lebt nicht mit Partner zusammen), das Alter (in Jahren sowie quadriert), Bildung (ISCED), die Einschätzung des Haushaltseinkommens (4 Stufen), ${ }^{6}$ ob Kinder in bestimmten Altersklassen im Haushalt leben (1-6 Jahre, 7-12 Jahre, 13-18 Jahre), die Betriebsgröße (1-9, 10-24, 25-99, 100-499 sowie 500 und mehr Beschäftigte) und der Sektor (= 1 Dienstleistungen; = 0 Verarbeitendes Gewerbe) getestet. Die herrschende Geschlechterkultur wird anhand von länderspezifischen Geschlechterrolleneinstellungen abgebildet. Im ESS stehen da-

\footnotetext{
4 Auf eine Unterteilung von Deutschland in Ost und West mussten wir, trotz der bekannten Unterschiede in Bezug auf Geschlechterrolleneinstellungen und der Integration von Frauen und Müttern in den Arbeitsmarkt, aufgrund der geringen Fallzahl in Ostdeutschland $(n=120)$ verzichten. Zwar werden, wie ausgeführt, regionale Unterschiede im Erwerbsverhalten von Frauen auf deskriptiver (Leibert 2014) und multivariater Ebene (Fernández 2012) auch für andere europäische Länder berichtet, es ist aufgrund der Fallzahlen in unserer Stichprobe jedoch nicht möglich, hierfür zu kontrollieren.

5 Zusätzlich haben wir kontrafaktische Analysen durchgeführt, um zu überprüfen, inwieweit die Bevölkerungsstruktur der einbezogenen Länder (Alter, Bildung) den Effekt der individuellen Charakteristika, insbesondere der Geschlechterrolleneinstellung, auf die aggregierten Arbeitszeiten beeinflussen könnte. Bei der Analyse orientierten wir uns an dem Vorgehen von Bick et al. (2018), indem wir die einzelnen Länder nach der Bevölkerungsstruktur Deutschlands gewichteten. Im Ergebnis bleibt der Effekt im Aggregat (mit Ausnahme von 4 Ländern) gleich: Frauen mit traditionellen Einstellungen haben im Durchschnitt kürzere Arbeitszeiten als Frauen mit progressiveren Einstellungen.

6 Eine aktuelle Studie von Bick et al. (2018) kommt zu dem Ergebnis, dass in Ländern mit einem hohen Einkommensniveau die Arbeitszeiten maßgeblich vom individuellen Stundenverdienst beeinflusst werden. Leider sind diese Angaben im ESS nicht für alle Länder vorhanden, weshalb wir hier auf die Berechnung dieses Effekts verzichten mussten.
} 
zu zwei Items zur Verfügung, aus denen wir einen 5-stufigen Index bilden: „Wenn Arbeitsplätze knapp sind, sollten Männer mehr Recht auf einen Arbeitsplatz haben als Frauen“ und „Eine Frau sollte dazu bereit sein, ihre Erwerbstätigkeit zu Gunsten ihrer Familie zu reduzieren“. Beide Items wurden umkodiert, sodass höhere Werte progressivere Einstellungen darstellen. Cronbachs Alpha liegt für das Sample bei 0,6 und weist damit einen, für einen aus nur zwei Items bestehenden Index, akzeptablen Wert auf (Cho und Kim 2015). ${ }^{7}$ Zwar weisen, wie ausgeführt, neuere Studien darauf hin, dass es sich bei Geschlechterrolleneinstellungen um ein multidimensionales Konstrukt handelt, was gegen die Verwendung eines einfachen Summenscores sprechen kann (Grunow et al. 2018; Knight und Brinton 2017; Yu und Lee 2013). Wir sind jedoch der Ansicht, dass die von uns verwendeten Items sich inhaltlich nah genug aufeinander beziehen, da beide Items die Wichtigkeit von Erwerbstätigkeit für Frauen thematisieren - zum einen im Vergleich zu Männern, zum anderen im Vergleich zu den Aufgaben in einer Familie. Tabelle 3 im Anhang zeigt die länderspezifischen Cronbachs-Alpha-Werte sowie die Mittelwerte und die Standardabweichungen der Variablen auf der Individualebene.

Bei den Makroindikatoren unterscheiden wir zwischen Variablen zu wohlfahrtsstaatlichen oder familienpolitischen Regelungen, Arbeitsplatzcharakteristika und soziostrukturellen Rahmenbedingungen. Die Daten stammen vorranging von Eurostat, OECD, WZB und dem European Company Survey (ECS) 2009. Eine genaue Übersicht der verwendeten Daten findet sich online unter http://www.iaq.uni-due. de/aktuell/veroeff/2016/Geschlechterrollen_Variablen.pdf. Bei wohlfahrtsstaatlichen oder familienpolitischen Maßnahmen fokussieren wir auf Kinderbetreuung, auf Elternzeitregelungen (Anzahl der bezahlten Wochen multipliziert mit der finanziellen Kompensierung) sowie auf die getrennte oder gemeinsame Besteuerung von Paaren. Zudem wird danach kontrolliert, ob Eltern einen Anspruch auf Kinderbetreuung haben und ob es im jeweiligen Land ein Recht auf Teilzeitarbeit gibt. Bei der Auswahl der Indikatoren orientieren wir uns an theoretischen Überlegungen sowie an Untersuchungen, die einen Zusammenhang zwischen wohlfahrtsstaatlichen oder familienpolitischen Maßnahmen und der Arbeitsmarktpartizipation von Frauen nachweisen konnten (z. B. Boeckmann et al. 2014; Jaumotte 2003; Pettit und Hook 2005; Uunk et al. 2005). Bei der Operationalisierung der Kinderbetreuung folgen wir, in Ermangelung besser geeigneter Daten, der Praxis, den Anteil an Kindern unter 3 Jahren, die in öffentlichen Kinderbetreuungseinrichtungen eingeschrieben sind, zu nutzen (Boeckmann et al. 2014; Budig et al. 2012; Hipp und Leuze 2015; Korpi et al. 2013). Dieses Vorgehen hat den Vorteil, dass die Daten für alle beobachteten Länder verfügbar und länderübergreifend vergleichbar sind. Ein Nachteil ist jedoch, dass das Vorhandensein von Kinderbetreuungsangeboten nur teilweise abgedeckt wird und informelle Regelungen nicht erfasst werden. Zudem kann die Nutzung von öffentlicher Kinderbetreuung ebenso eine Folge der Erwerbstätigkeit von Frauen sein, anstatt der Ursache (Boeckmann et al. 2014). Bei den Arbeitsplatzcharakteristika testen wir, inwieweit die Verbreitung von flexiblen Arbeitszeitformen auf Betriebsebene die Länge der Erwerbsarbeitszeiten von Frauen beeinflusst. Dabei dient dieser

\footnotetext{
7 Dies vor dem Hintergrund, dass Cronbachs Alpha von der Testlänge abhängig ist (vgl. u. a. Cho und Kim 2015, S. 215).
} 
Wert als ein Proxy für die Familienfreundlichkeit der Betriebe. Einerseits nutzen wir Angaben der Arbeitgeberseite, ob Beschäftigte (a) in ihrem Betrieb die Möglichkeit haben, Arbeitszeitkonten zu nutzen, und (b) ob ihr Betrieb Beschäftigten die Möglichkeit bietet, innerhalb gewisser Grenzen die Zeit des täglichen Arbeitsbeginns und Arbeitsendes den persönlichen Bedürfnissen oder Wünschen anzupassen (Angebot). Zusätzlich verwenden wir einen Indikator, bei dem Arbeitnehmervertretungen gefragt werden, ob in ihrem Betrieb Beschäftigte flexible Arbeitszeitformen nutzen (Nutzung). Die Variablen stammen aus dem European Company Survey 2009 und wurden aggregiert, sodass wir Länderanteile auf Ebene 2 in die Analyse aufnehmen können.

Darüber hinaus prüfen wir mögliche Effekte weiterer arbeitsmarktbezogener und soziostruktureller Variablen, wie den Einfluss der Arbeitslosenquote, weiblicher Beschäftigungsquote, geschlechtsspezifischer Unterschiede in der Beschäftigtenquote, Wirtschaftswachstum etc. Um die Modellgüte zu erhöhen, wurden insignifikante unabhängige Variablen aus dem Modell entfernt (Braun et al. 2010; Hans 2006). Eine vollständige Auflistung der überprüften, aber nichtsignifikanten Variablen findet sich online unter http://www.iaq.uni-due.de/aktuell/veroeff/2016/ Geschlechterrollen_Variablen.pdf (Tab. B).

Die abhängige Variable bilden die normalerweise in der Woche geleisteten Arbeitsstunden: „Wie viele Stunden arbeiten Sie normalerweise tatsächlich in einer Woche in Ihrem Hauptberuf, einschließlich bezahlter oder unbezahlter Überstunden“ (ESS). Aus Plausibilitätsgründen wurden Arbeitsstunden ab $80 \mathrm{~h}$ pro Woche gekappt.

\section{Ergebnisse}

Für die Überprüfung unserer Hypothesen berechnen wir ein Random Slope-Modell, in dem die Varianzparameter der Geschlechterrolleneinstellung freigesetzt und somit als länderspezifisch modelliert werden und operationalisieren so Pfau-Effingers Konzept der Geschlechterkulturen. Alle unabhängigen Variablen bis auf DummyVariablen werden an ihrem Mittelwert in der gesamten Stichprobe zentriert. Dieses Vorgehen hat mehrere Vorteile (Braun et al. 2010). Erstens erhalten zentrierte unabhängige Variablen (u. a. Alter, Betriebsgröße) dadurch einen plausiblen Nullpunkt. Zweitens kann die Konstante als kontextübergreifender Schätzwert für Personen, die bei den individuellen Faktoren mittlere Ausprägungen besitzen, interpretiert werden. Drittens kann sich die Kovarianz zwischen den Varianzkomponenten der Konstanten und der Regressionskoeffizienten dadurch verändern und somit Multikollinearitätsproblemen entgegenwirken. ${ }^{8}$ Die Ergebnisse unserer Berechnungen sind in Tab. 2 dargestellt.

\footnotetext{
${ }^{8}$ Um unsere Hypothesen zu überprüfen, sind wir mehrstufig vorgegangen und haben individuelle Merkmale, kontextuelle Faktoren und Cross-Level-Interaktionen nacheinander eingeführt. Die Ergebnisse der einzelnen Modelle sind von der Autorin zu erhalten: http://www.iaq.uni-due.de/aktuell/veroeff/2019/ Geschlechterrollen_entscheidend_Tabellenanhang.pdf.
} 
Tab. 2 Ergebnisse der Mehrebenanalyse (finales Modell) - Determinanten weiblicher Erwerbsarbeitszeit

\begin{tabular}{|c|c|c|c|}
\hline & \multicolumn{3}{|l|}{ Modell 3} \\
\hline & Coef & SE & Datenquelle \\
\hline Konstante & $38,80 * * *$ & 0,53 & - \\
\hline \multicolumn{4}{|l|}{ Individuelle Merkmale } \\
\hline Partner im $\mathrm{HH}$ & $-0,66 * *$ & 0,26 & ESS \\
\hline Kinder im HH (1-6 J.) & $-1,65^{*}$ & 0,68 & ESS \\
\hline Kinder im HH (7-12 J.) & $-1,97 * * *$ & 0,59 & ESS \\
\hline Kinder im HH (13-18 J.) & $-1,42 * * *$ & 0,39 & ESS \\
\hline Alter & $0,64 * * *$ & 0,12 & ESS \\
\hline Alter quadriert & $-0,01 * * *$ & 0,00 & ESS \\
\hline Bildung (ISCED) & $0,44^{*}$ & 0,20 & ESS \\
\hline Einschätzung Haushaltseinkommen & $0,95 * * *$ & 0,16 & ESS \\
\hline Geschlechterrolleneinstellung & $0,80 * * *$ & 0,19 & ESS \\
\hline \multicolumn{4}{|l|}{ Betriebliche Faktoren } \\
\hline Betriebsgröße & $0,85 * * *$ & 0,13 & ESS \\
\hline Dienstleistungsgewerbe & $-1,81 * * *$ & 0,40 & ESS \\
\hline \multicolumn{4}{|l|}{ Kontextfaktoren } \\
\hline Kinderbetreuung & $0,13 * * *$ & 0,02 & Eurostat \\
\hline Inanspruchnahme von flexiblen Arbeitszeitformen & $-0,14 * * *$ & 0,03 & ECS \\
\hline Gleitzeit & $-0,16^{* *}$ & 0,08 & ECS \\
\hline Elternzeitregelungen & $-0,07$ & 0,08 & Eurostat \\
\hline Gemeinsame Besteuerung & $-1,60 * *$ & 0,65 & OECD \\
\hline \multicolumn{4}{|l|}{ Cross-Level-Effekte } \\
\hline Kinderbetreuung * Geschlechterrolleneinstellung & $0,02 *$ & 0,01 & - \\
\hline $\begin{array}{l}\text { Inanspruchnahme von flexiblen Arbeitszeitformen * } \\
\text { Geschlechterrolleneinstellung }\end{array}$ & $0,03^{+}$ & 0,02 & - \\
\hline Kinder im HH (1-6 J.) * Elternzeitregelungen & $0,18^{*}$ & 0,09 & - \\
\hline Kinder im HH (7-12 J.) * Elternzeitregelungen & $0,17 *$ & 0,08 & - \\
\hline Kinder im HH (13-18 J.) * Elternzeitregelungen & $0,18 * * *$ & 0,04 & - \\
\hline$I C C$ & 0,05 & & - \\
\hline Devianz & 60.981 & & - \\
\hline$N$ & 8268 & & - \\
\hline
\end{tabular}

Referenzkategorien: Keine Kinder, Verarbeitendes Gewerbe, Individuelle Besteuerung

In die Analyse gingen folgende Länder ein: BE, BG, CY, CZ, DE, DK, EE, ES, FI, FR, GR, HU, IE, LT, LV, NL, PL, PT, RO, SE, SI, SK, UK

ICC: 0,13 ;

$* * * p<0,001, * * p<0,01, * p<0,05,{ }^{+} p<0,1$

Die Berechnung des Nullmodells, das nur die zufällig variierende Regressionskonstante enthält, ergibt über alle Befragten und Länder hinweg eine mittlere Arbeitszeit von Frauen von 37,21 h. Die Intraklassenkorrelation liegt bei 0,13; das heißt, dass länderspezifische Faktoren $13 \%$ der Gesamtvarianz erklären können. Somit stellt die Mehrebenenanalyse eine adäquate Modellierungsstrategie dar.

Wir betrachten zunächst die Kontrollvariablen, deren Koeffizienten sämtlich in die aus der Literatur erwartete Richtung weisen. So zeigen unsere Analysen, dass das 
Lebensalter die Dauer der Erwerbsarbeitszeit zunächst positiv beeinflusst $(p<0,001)$, sich dieser Effekt jedoch im höheren Alter umkehrt $(p<0,001)$. Je höher die Bildung der befragten Frauen, desto höher auch ihre wöchentliche Erwerbsarbeitszeit $(p<0,05)$. Frauen, die im Dienstleistungsgewerbe beschäftigt sind, haben kürzere Erwerbsarbeitszeiten als Frauen im Verarbeitenden Gewerbe $(p<0,001)$, zudem arbeiten Frauen in größeren Betrieben durchschnittlich länger als Frauen in kleineren Betrieben. Außerdem wirkt sich die subjektive Wahrnehmung des Haushaltseinkommens auf die Länge der Erwerbsarbeitszeiten aus: Je besser Frauen ihr Haushaltseinkommen einschätzen, desto kürzer sind ihre Erwerbsarbeitszeiten. Dies ist ein Indikator dafür, dass sich Frauen in ihren Erwerbsarbeitszeitentscheidungen an ökonomischen Gesichtspunkten orientieren. Ist der Haushalt gut situiert, ,leisten“ sie sich, einen geringeren Umfang an Erwerbsarbeitszeit zu realisieren.

Im Folgenden analysieren wir unsere Ergebnisse vor dem Hintergrund der von uns aufgestellten Hypothesen. Hypothese 1, nach der der Umfang der Erwerbsarbeitszeiten von Frauen signifikant davon beeinflusst wird, ob sie mit einem Partner und/oder Kindern zusammenleben, wird durch unsere Analysen bestätigt. Frauen, die mit ihrem Partner in einem Haushalt leben, haben kürzere Erwerbsarbeitszeiten $(p<0,05)$ als Frauen ohne Partner (im Haushalt). Auch Kinder wirken sich für Frauen negativ auf den Umfang der geleisteten Erwerbsarbeitszeit aus $(p<0,05)$. Bezüglich der länderspezifischen Effekte der Geschlechterrolleneinstellung zeigt sich ein positiver Zusammenhang zwischen der Geschlechterrolleneinstellung und der Erwerbsarbeitszeit $(p<0,001)$. Damit bestätigt sich unsere Hypothese 2.

Hypothese 3 (soziostrukturelle Rahmenbedingungen wirken sich auf die Arbeitszeiten von Frauen aus) wird durch unsere Analyse nur teilweise bestätigt. So hat zwar, wie vermutet, die Nutzung öffentlicher Kinderbetreuung einen positiven Effekt auf den Umfang der Erwerbsarbeitszeiten von Frauen $(p<0,001)$ (Hypothese 3a), während sich die gemeinsame Besteuerung von verheirateten Paaren negativ auswirkt $(p<0,05)$ (Hypothese 3c). Der postulierte positive Effekt der Elternzeit lässt sich auf Basis unserer Analysen jedoch nicht nachweisen, der Koeffizient bleibt insignifikant (Hypothese 3b).

Auch Hypothese 4, nach der das Vorhandensein vereinbarkeitsförderlicher Arbeitszeitorganisation sich positiv auf die Länge der Erwerbsarbeitszeit von Frauen auswirkt, muss auf Basis unserer Daten zunächst verworfen werden. In den Ländern, in denen Gleitzeit weit verbreitet ist, ist die Erwerbsarbeitszeit von Frauen kürzer als in Ländern, in denen das Angebot an Gleitzeit gering ist $(p<0,01)$ (Hypothese 4a). Der gleiche Effekt findet sich hinsichtlich der Arbeitszeitsouveränität (Hypothese 4b). In den Ländern, in denen die Inanspruchnahme flexibler Erwerbsarbeitszeiten hoch ist, sind die wöchentlichen Erwerbsarbeitszeiten von Frauen geringer $(p<0,001)$. Eine mögliche Erklärung für die negativen Effekte der flexiblen Arbeitszeitformen als auch für den insignifikanten Effekt der Elternzeit liefert das sogenannte Wohlfahrtsstaatparadoxon (Mandel und Semyonov 2006). Es besagt, dass die Förderung der Erwerbsbeteiligung von Frauen nicht immer zu den intendierten Folgen führt: Sozialdemokratische Wohlfahrtsregimes fördern die Arbeitsmarktpartizipation von Frauen durch familienpolitische Maßnahmen (z.B. die Bereitstellung von öffentlicher Kinderbetreuung oder die Gewährung von Elternzeit) oder familienfreundliche Beschäftigungspraktiken (z.B. flexible Arbeitszeitformen), allerdings 
auf Kosten geschlechtsspezifischer sektoraler und beruflicher Segregation sowie eingeschränkten Zugangs zu günstigen Positionen und Teilzeitarbeit. Im Gegensatz dazu wird die Arbeitsmarktpartizipation von Frauen oder Müttern in liberalen Regimes weder explizit einschränkt noch gefördert. Konservative Wohlfahrtsregimes hingegen benachteiligen Frauen in beiden Aspekten (Mandel und Semyonov 2006). Eine weitere mögliche Erklärung könnte auch darin liegen, dass flexible, mit Arbeitszeitsouveränität verbundene, Arbeitszeitmodelle vor allem in Ländern angeboten werden, die im Durchschnitt geringere Erwerbsarbeitszeiten aufweisen (Eurofound 2015).

Mittels der Berechnung von Cross-Level-Effekten überprüfen wir Hypothese 4 (das Ausmaß, in dem familienpolitische Leistungen und eine vereinbarkeitsförderliche Arbeitszeitorganisation die Dauer der Erwerbsarbeitszeiten von Frauen beeinflussen, wird von länderspezifischen Geschlechterrolleneinstellungen determiniert). Die Analysen bestätigen unsere Hypothese: Die ebenenübergreifenden Interaktionen zwischen Kinderbetreuung und der länderspezifischen Geschlechterrolleneinstellung $(p<0,05)$ sowie zwischen der Nutzung flexibler Erwerbsarbeitszeitformen und Geschlechterrolleneinstellung $(p<0,07)$ sind positiv. Das heißt, die länderspezifischen Geschlechterkulturen wirken sich auf den Effekt zwischen unabhängiger und abhängiger Variable aus. Da wir jedoch keinen Schluss über die kausale Richtung treffen können, lassen sich die Ergebnisse in zweierlei Weise interpretieren: Erstens könnte die Inanspruchnahme von öffentlichen Kinderbetreuungseinrichtungen bei den Frauen den Umfang der Erwerbsarbeitszeit erhöhen, wenn die länderspezifische Geschlechterrolleneinstellung progressiv ist. Zweitens fallen länderspezifische Geschlechterrolleneinstellungen je nach Höhe der Inanspruchnahme von Kinderbetreuung bei den Individuen stärker oder schwächer ins Gewicht, wenn es um die Dauer der Erwerbsarbeitszeiten geht. Denselben Effekt können wir bei der Interaktion zwischen der Nutzung flexibler Arbeitszeitformen und Geschlechterrolleneinstellung feststellen, obzwar dieser nur schwach signifikant ist. Zusätzlich interagieren wir Elternzeitregelungen (Länge der Elternzeit gewichtet nach der finanziellen Unterstützung) mit dem Vorhandensein von Kindern im Haushalt. Dabei zeigt sich ein positiver Zusammenhang zwischen der Interaktion und der Höhe der Erwerbsarbeitszeit von Frauen $(p<0,05)$. Das heißt, in Ländern mit längerer finanziell kompensierter Elternzeit arbeiten Frauen mit Kindern mehr Stunden als in Ländern mit kürzerer Elternzeit. Die Signifikanz dieser Effekte variiert zwischen jüngeren und älteren Kindern im Haushalt, wobei der Effekt für ältere stärker ausfällt. Eine mögliche Erklärung ist, dass eine Erwerbstätigkeit von Frauen mit älteren Kindern ohnehin akzeptierter ist und damit vorherrschende moderne Elternzeitregelungen im betroffenen Land hier eher einen größeren Hebeleffekt haben könnten als bei sehr kleinen Kindern. Hinzu kommt möglicherweise ein technischer Effekt: Je nach zeitlicher Abgrenzung der Mikrodaten und der verwendeten Makrodaten zur Elternzeit kann die Situation auftreten, dass Eltern kleiner Kinder von der Leistung gegebenenfalls noch gar nicht profitieren konnten. 


\section{Zusammenfassung und Diskussion}

Die Erwerbsarbeitszeiten von Frauen variieren deutlich im internationalen Vergleich, während in einigen Ländern (darunter Deutschland, die Niederlande und Österreich) Frauen und insbesondere Mütter nur über einen vergleichbar geringen wöchentlichen Stundenumfang in das Erwerbsleben eingebunden sind, liegen ihre durchschnittlichen Erwerbsarbeitszeiten in den osteuropäischen und Nordischen Ländern deutlich höher. Da berufliche Entwicklungsmöglichkeiten, finanzielle Unabhängigkeit und die im Alter zu erwartende Rente eng mit der Dauer der durchschnittlich geleisteten Erwerbsarbeitszeit zusammenhängen, war es das Ziel unserer Studie, diejenigen Determinanten zu bestimmen, die dazu beitragen, den Umfang der durchschnittlich geleisteten Erwerbsarbeitszeiten von Frauen und insbesondere Müttern zu erhöhen.

Auf der Theorie der Geschlechterkultur von Pfau-Effinger (1996) und den Arbeiten von Macdonald (2009) aufbauend, gingen wir davon aus, dass der Umfang der von Frauen geleisteten Erwerbsarbeitszeiten das Resultat eines komplexen Zusammenspiels individueller und makrostruktureller Faktoren ist, deren Wirkung durch die in einem Land dominierende herrschende Geschlechterkultur beeinflusst wird. Zentral ist dabei die Annahme, dass (Erwerbsarbeitszeit-)Entscheidungen und Handeln keine bloße Reaktion auf soziostrukturelle Bedingungen oder ihre Veränderung sind, sondern diese auch ein Produkt von geteilten Normen und Vorstellungen über eine geeignete Aufgabenteilung der Geschlechter darstellen. Um diese These zu überprüfen, nutzten wir Daten des ESS und entwickelten ein Mehrebenendesign, indem erstmalig Geschlechterkultur nicht nur als zusätzlich erklärende, sondern auch als mit Makrostrukturen interagierende Variable in die Gleichung eingefügt wurde. Dieses Vorgehen erlaubte uns, die von Pfau-Effinger (1996) entwickelte Theorie der Geschlechterkulturen auf unsere Fragestellung anzuwenden und empirisch zu überprüfen. Unsere Analysen zeigen, dass sich Pfau-Effingers Annahmen über die Rolle von Geschlechterkulturen bestätigen lassen: Erwerbsarbeitszeiten von Frauen basieren auf einem komplexen Zusammenspiel individueller und makrostruktureller Faktoren. Dabei wird die Wirkung makrostruktureller Maßnahmen maßgeblich von der Ausprägung gesellschaftlich geteilter Vorstellungen beeinflusst.

Auf der individuellen Ebene ergeben unsere Berechnungen, dass über alle Länder hinweg der Umfang der Erwerbsarbeitszeiten von Frauen durch die Anwesenheit eines Partners und Kindern im Haushalt negativ beeinflusst wird (Hypothese 1). Außerdem bestätigen sie die Annahme, dass der von Frauen geleistete Erwerbsarbeitszeitumfang mit der in einem Land herrschenden Geschlechterkultur zusammenhängt (Hypothese 2): In Ländern mit einer progressiven Geschlechterkultur weisen Frauen durchschnittlich höhere Erwerbsarbeitszeiten auf als in Ländern, in denen die Geschlechterkultur weniger progressiv ist. Die Effekte, die wir bezüglich der Wirkung struktureller Rahmenbedingungen feststellen, stimmen dagegen nur partiell mit unseren Hypothesen überein. Hinsichtlich der erwarteten Einflüsse familienpolitischer Leistungen auf die Dauer der Erwerbsarbeitszeiten finden wir zunächst, dass sich die Möglichkeit, öffentliche Kinderbetreuungseinrichtungen nutzen zu können, erwerbsarbeitszeitverlängernd auswirkt (Hypothese 3a). Auch zeigt sich in unseren Analysen der erwartete negative Effekt einer gemeinsamen steuerlichen Veranlagung von Paaren auf die Erwerbsarbeitszeiten von Frauen (Hypothese 3c). Anzunehmen 
ist, dass dieser Effekt doppelt durchschlägt, wenn in Ländern, wie z. B. in Deutschland, gleichzeitig Regelungen wie der Mini-Job zu finden sind, die Verdienste unter einer gewissen Grenze steuerfrei stellen.

Entgegen unseren Annahmen konnten wir weder für die Elternzeit (Hypothese $3 b$ ) noch für die vereinbarkeitsförderliche Arbeitszeitorganisation (Hypothese 4a und 4b) positive Haupteffekte auf den Umfang der Erwerbsarbeitszeiten feststellen. Neben methodischen und technischen Erwägungen (vgl. den vorherigen Abschnitt) lassen sich diese Ergebnisse unseres Erachtens zumindest teilweise mit dem Wohlfahrtsstaatsparadoxon (Mandel und Semyonov 2006) erklären. Zwar beziehen sich die Autoren in ihren Ausführungen auf die berufliche Segregation und Unterrepräsentation von Frauen in Leitungspositionen, sie lassen sich aber gut auf die vorliegenden Ergebnisse übertragen. Demnach wirken (familienpolitische) Leistungen und Maßnahmen, die sich positiv auf die Vereinbarkeit von Berufstätigkeit und familiären Verpflichtungen auswirken sollen, nicht genderneutral und können einen Bumerangeffekt erzielen, indem sie geschlechtsspezifische Rollen- und Aufgabenzuweisungen manifestieren. In der Folge wirken sich diese Maßnahmen zwar positiv auf die Erwerbstätigkeit von Frauen per se aus. Da aber die Rolle der Frau als primär Verantwortliche für Kinder und Haushalt nicht infrage gestellt wird, bleibt ihre Berufstätigkeit häufig, sowohl zeitlich als auch im Hinblick auf das Verfolgen einer eigenen Karriere, beschränkt.

Die Wichtigkeit der Rolle von länderspezifischen Geschlechterkulturen für das Ausmaß, in dem makrostrukturelle Gegebenheiten sich im Handeln von Individuen widerspiegeln, wird durch die erhaltenen signifikanten Cross-Level-Interaktionen belegt. Damit bestätigt sich unsere Hypothese, dass sich der Grad, in dem soziostrukturelle und betriebliche Rahmenbedingungen auf die Dauer der Erwerbsarbeitszeiten von Frauen auswirken, von der in einem Land herrschenden Geschlechterkultur beeinflusst wird (Hypothese 4). Dieses Ergebnis zeigt unseres Erachtens deutlich, dass der Erfolg von vereinbarkeitsfördernden Maßnahmen - unabhängig davon, ob sie betrieblich oder staatlich initiiert sind - von den in einer Gesellschaft geteilten Vorstellungen über die geschlechtsspezifische Aufgabenverteilung abhängig ist. Diese Angebote können dann zu längeren Erwerbsarbeitszeiten von Frauen führen, wenn die herrschende Geschlechterkultur eine stärkere Einbindung von Frauen und insbesondere Müttern in das Erwerbsleben als normenkonform ,erlaubt“. Umgekehrt laufen solche Maßnahmen Gefahr, nicht ihre volle Wirkung zu entfalten oder gar zu verpuffen, wenn die in einem Land herrschende Geschlechterkultur eher traditionell geprägt ist oder beispielsweise egalitäre Vorstellungen zur Erwerbstätigkeit von Frauen und Müttern sowie essenzialistische Einstellungen, die die Unterschiede zwischen Männern und Frauen betonen, parallel auftreten. So zeigt die Studie von Yu und Lee (2013), dass in Ländern mit egalitäreren Strukturen Befragte die Berufstätigkeit von Müttern zwar befürworten, eine gleichberechtigte Arbeitsteilung im Haushalt aber eher ablehnen. Übertragen auf unsere Fragestellung würde das bedeuten, dass Frauen zwar zunehmend häufiger eine Erwerbstätigkeit aufnehmen, der Stundenumfang jedoch limitiert bleibt, um nicht in Konflikt mit häuslichen Verpflichtungen zu kommen. Vor diesem Hintergrund ist es ein Nachteil unserer Studie, dass wir aufgrund der Datenlage, die in einem Land herrschenden Geschlechterrollenvorstellungen nicht in ihrer ganzen mehrdimensionalen Komplexität abbilden 
haben können (Grunow et al. 2018; Knight und Brinton 2017). Der von uns verwendete ESS erfasst Geschlechterrolleneinstellungen nur anhand zweier Items und lässt deshalb eine multidimensionale Modellierung nicht zu. Allerdings halten wir diesen Ansatz für Fragestellungen, die sich, wie die unsere, mit dem Zusammenhang makrostruktureller Gegebenheiten und individuellem Verhalten beschäftigten, für die Zukunft vielversprechend, da er genauere Hinweise dafür liefern kann, warum welche Arten von soziostrukturellen Veränderungen in einigen Ländern den intendierten Erfolg haben und in anderen Ländern nicht.

Damit eng verbunden ist die Frage der Persistenz von Geschlechterkulturen. In unserem Beitrag behandeln wir Länder als bezüglich der Geschlechterkultur homogene Einheiten, was, wie beschrieben, eine der Datenlage geschuldete Vereinfachung darstellt. Wahrscheinlicher ist jedoch, dass auch innerhalb eines Landes sowohl intergenerational als auch regional unterschiedlich ausgeprägte Geschlechterkulturen existieren (vgl. Yu und Lee 2013). Hinweise dazu gibt es z. B. für Deutschland (OstWest-Vergleich) oder in Italien (Nord-Süd-Vergleich). Eine stärkere Berücksichtigung von länderinternen Unterschieden könnte die Aussagekraft unseres Modells noch verstärken.

Da unsere Analysen auf Individual- und Querschnittsdaten beruhen, ist ihre Aussagekraft naturgemäß eingeschränkt. Querschnittsdaten stellen immer nur Momentaufnahmen dar und lassen deshalb keine Aussagen über Entwicklungen, Pfadabhängigkeiten oder Kausalitäten zu. Offene Fragen sind daher unter anderen, inwieweit sich Makrostruktur und Geschlechterkultur gegenseitig beeinflussen und in welchem zeitlichen Rahmen gegebenenfalls Anpassungen vonstattengehen, oder auch wie manifest und änderungsresistent (welche) Geschlechterkulturen im Zeitverlauf sind. Nicht vollständig klären können wir auch die Tatsache, dass auch Frauen ohne Kinder, oder deren Kinder nicht mehr im selben Haushalt leben, signifikant kürzere Arbeitszeiten als Männer aufweisen.

Zudem bleibt in unserer Analyse die konkrete Haushaltssituation außen vor. Über Erwerbsstatus, Qualifikation und die beruflichen Umstände des Partners (wenn im Haushalt vorhanden) sowie der jeweilige Beitrag zum Haushaltseinkommen haben wir keine Information vorliegen. Dabei ist davon auszugehen, dass realisierte Erwerbsarbeitszeiten in Paarhaushalten, insbesondere wenn auch Kinder vorhanden sind, immer auch eine Funktion der relativen Ressourcen und Potenziale beider Partner sind (vgl. Hipp und Leuze 2015). Hier besteht also noch weiterer Forschungsbedarf.

Trotz der genannten Einschränkungen sind wir der Ansicht, mit unserer Studie einen wichtigen Beitrag für unsere Fragestellung liefern zu können. So konnten wir mit unserer Arbeit zeigen, dass sich die theoretischen Annahmen Pfau-Effingers (1996) über das Zusammenspiel von Geschlechterkulturen und Makrostrukturen in ein Mehrebenenmodell übertragbar und damit empirisch überprüfbar machen lassen. Außerdem liefert unsere Analyse Hinweise darauf, dass politische Maßnahmen, die sich auf eine bessere Einbindung von Frauen in den Arbeitsmarkt beziehen, nur dann ihre volle Wirksamkeit entfalten können, wenn sie durch die in einer Gesellschaft vorherrschenden Rollen- und Familienleitbilder gestützt werden - was notwendigerweise auch eine Veränderung der Rollenvorstellungen für Männer miteinschließt. 
Danksagung Wir danken den Gutachter*innen und Herausgeber*innen für Ihre wertvollen Hinweise und Kommentare.

Förderung Das Projekt wurde von der Hans-Böckler Stiftung gefördert.

Funding Open Access funding provided by Projekt DEAL.

Open Access Dieser Artikel wird unter der Creative Commons Namensnennung 4.0 International Lizenz veröffentlicht, welche die Nutzung, Vervielfältigung, Bearbeitung, Verbreitung und Wiedergabe in jeglichem Medium und Format erlaubt, sofern Sie den/die ursprünglichen Autor(en) und die Quelle ordnungsgemäß nennen, einen Link zur Creative Commons Lizenz beifügen und angeben, ob Änderungen vorgenommen wurden.

Die in diesem Artikel enthaltenen Bilder und sonstiges Drittmaterial unterliegen ebenfalls der genannten Creative Commons Lizenz, sofern sich aus der Abbildungslegende nichts anderes ergibt. Sofern das betreffende Material nicht unter der genannten Creative Commons Lizenz steht und die betreffende Handlung nicht nach gesetzlichen Vorschriften erlaubt ist, ist für die oben aufgeführten Weiterverwendungen des Materials die Einwilligung des jeweiligen Rechteinhabers einzuholen.

Weitere Details zur Lizenz entnehmen Sie bitte der Lizenzinformation auf http://creativecommons.org/ licenses/by/4.0/deed.de. 


\section{Anhang}

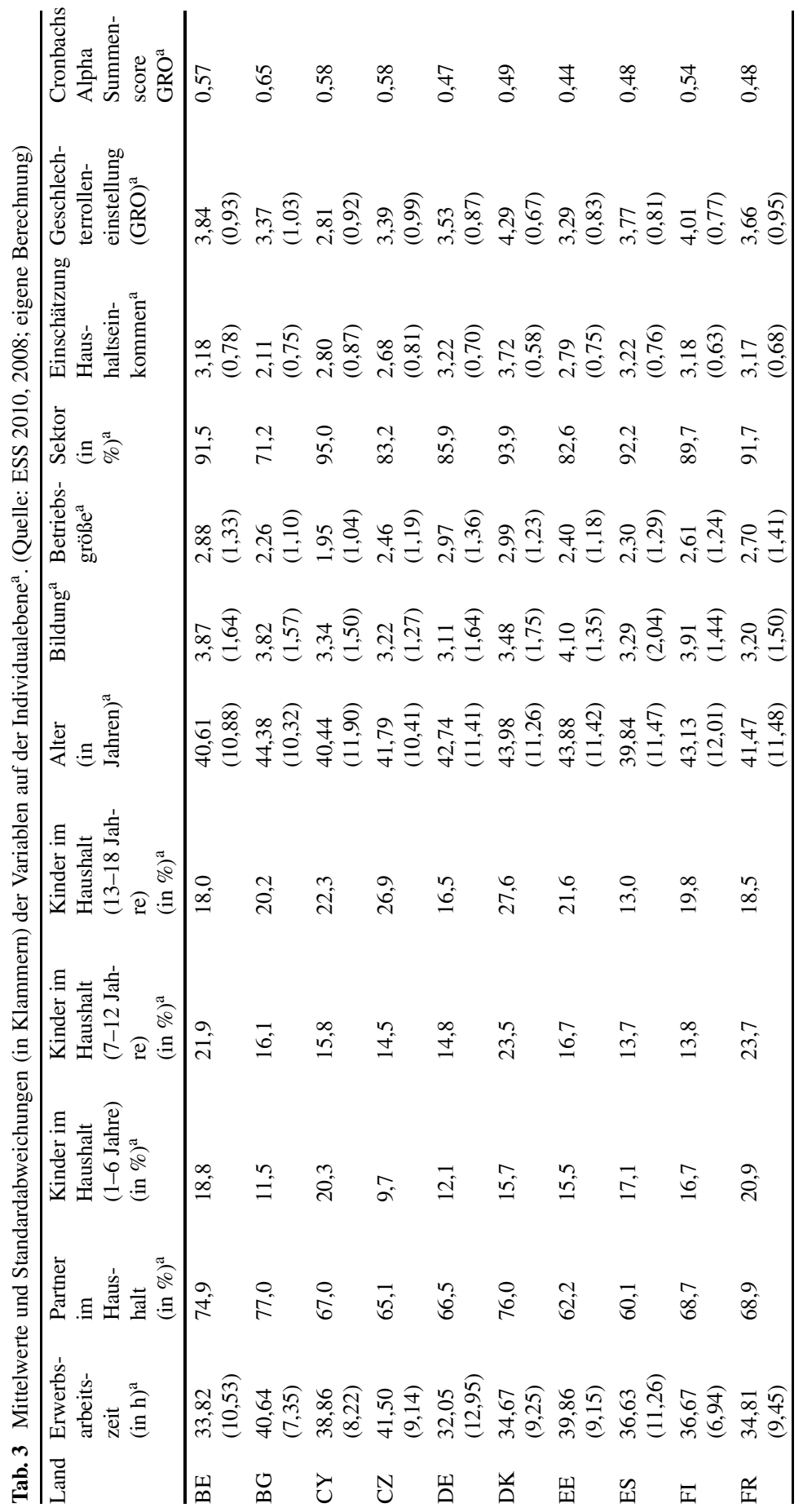




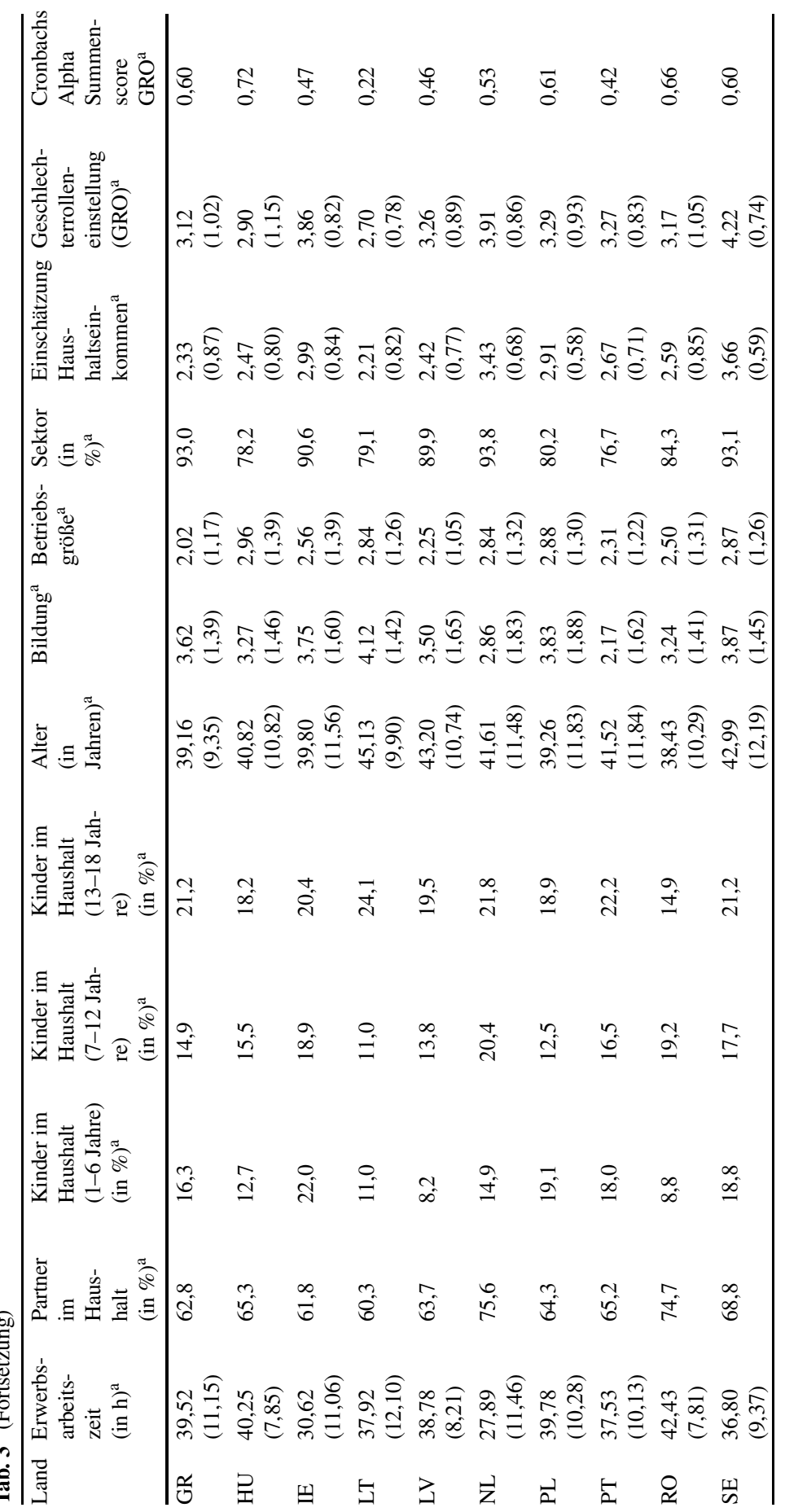




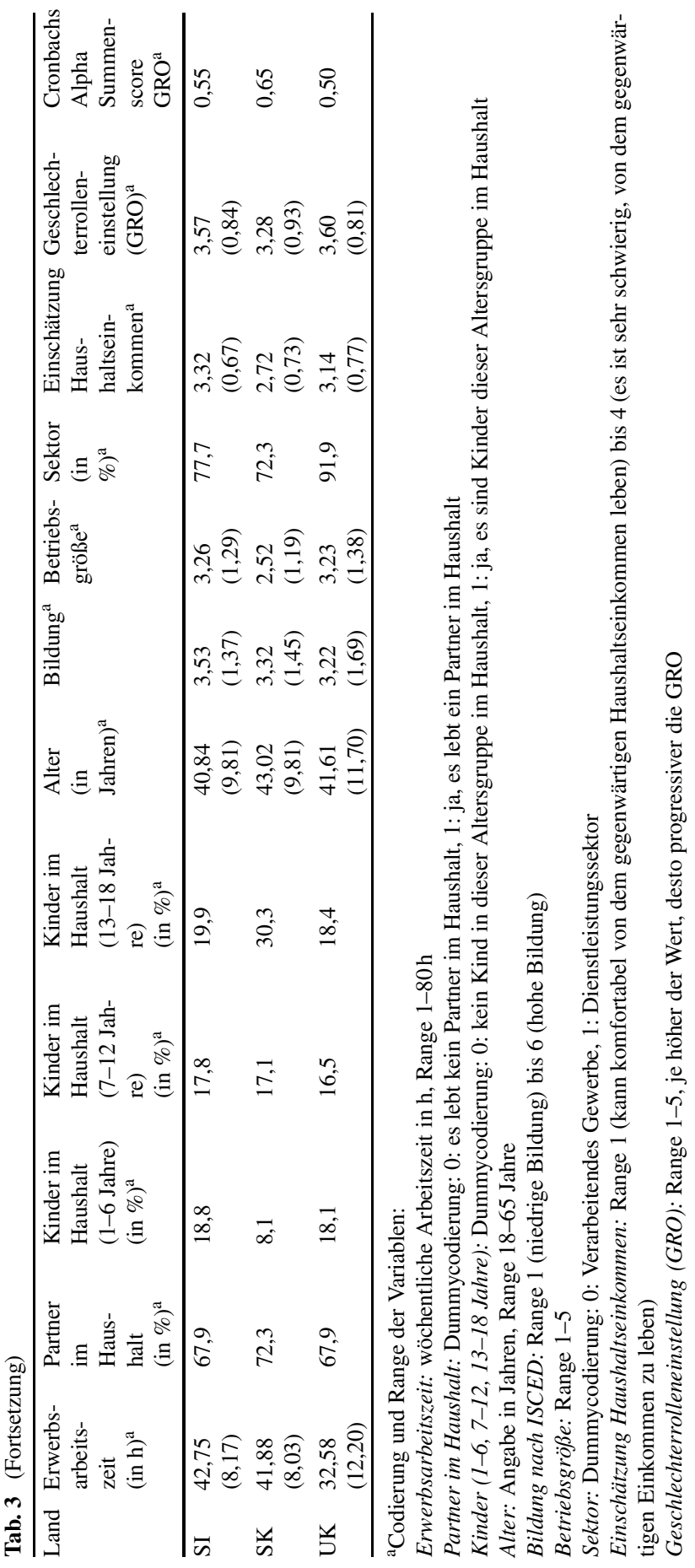




\section{Literatur}

Alfermann, Dorothea. 1996. Geschlechterrollen und geschlechtstypisches Verhalten. Stuttgart: Kohlhammer.

Allmendinger, Jutta, und Julia Haarbrücker. Mitarbeit Florian Fliegner. 2013. Lebensentwürfe heute - Wie junge Frauen und Männer in Deutschland leben wollen. Kommentierte Ergebnisse der Befragung 2012. WZB Discussion Paper P2013-002. https://bibliothek.wzb.eu/pdf/2013/p13-002.pdf (Zugegriffen: 21. Aug. 2019).

Anxo, Dominique, Colette Fagan, Inmaculada Cebrian und Gloria Moreno. 2007. Patterns of labour market integration in Europe-a life course perspective on time policies. Socio-Economic Review 5:233-260.

Anxo, Dominique, Christine Franz und Angelika Kümmerling. 2013. Working time and work-life balance in a life course perspective. A report based on the fifth European Working Conditions Survey. Project: Fifth European Working Conditions Survey. Dublin: Eurofound (2012).

Attanasio, Orazio, Hamish Low und Virginia Sanchez-Marcos. 2008. Explaining Changes in Female Labor Supply in a Life-Cycle Model. American Economic Review 98:1517-1552.

Becker, Gary Stanley. 1985. Human Capital, Effort, and the Sexual Division of Labor. Journal of Labor Economics 3:33-58.

Bick, Alexander, Nicola Fuchs-Schündeln und David Lagakos. 2018. How Do Hours Worked Vary with In-come? Cross-Country Evidence and Implications. American Economic Review 108(1):170-199.

BMFSFJ (Bundesministerium für Familie, Senioren, Frauen und Jugend). 2011. Neue Wege - Gleiche Chancen. Gleichstellung von Frauen und Männern im Lebensverlauf. Erster Gleichstellungsbericht. https://www.bmfsfj.de/blob/jump/93682/erster-gleichstellungsbericht-neue-wege-gleiche-chancendata.pdf (Zugegriffen: 21. Aug. 2019).

Boeckmann, Irene, Joya Misra und Michelle J. Budig. 2014. Mother's employment in wealthy countries. Cultural and Institutional factors shaping mother's employment and working hours in postindustrial countries. Social Forces 93:1301-1333.

Boll, Christina. 2014. Erwerbstätigkeit und Einkommen von Frauen im europäischen Vergleich - stilisierte Fakten und politische Implikationen. NZA - Neue Zeitschrift für Arbeitsrecht Beilage 2/2014 (zu Heft 22/2014):75-81.

Boll, Christina, Julian Leppin und Nora Reich. 2011. Einfluss der Elternzeit von Vätern auf die familiale Arbeitsteilung im internationalen Vergleich. Studie im Auftrag des Bundesministeriums für Familie, Senioren, Frauen und Jugend (BMFSFJ). HWWI Policy Paper 59.

Booth, Alison L., und Jan C. van Ours. 2010. Part-Time Jobs: What Women Want? CentER Discussion Paper Series 2010-05.

Braun, Daniela, Nicole Seher, Markus Tausendpfund und Ansgar Wolsing. 2010. Einstellungen gegenüber Immigranten und die Zustimmung zur Europäischen Integration - Eine Mehrebenenanalyse. Mannheimer Zentrum für Europäische Sozialforschung: Arbeitspapiere 136.

Brenke, Karl. 2015. Wachsende Bedeutung von Frauen auf dem Arbeitsmarkt. DIW Wochenbericht 5:75-86.

Budig, Michelle J., Joya Misra und Irene Böckmann. 2012. The Motherhood Penalty in Cross-National Perspective: The Importance of Work-Family Policies and Cultural Attitudes. Social Politics 19:163-193.

Campa, Pamela, Alessandra Casarico und Paola Profeta. 2011. Gender culture and gender gap in employment. CESifo Economic Studies 57:157-182.

Cho, Eunseong, und Seonghoon Kim. 2015. Cronbachs Coefficient Alpha. Well Known but Poorly Understood. Organizational Research Methods 18:1-24.

Corrigal, Elizabeth A., und Alison M. Konrad. 2007. Gender role attitudes and careers. A longitudinal study. Sex roles 56:847-855.

Dex, Shirley, und Heather Joshi. 1999. Careers and motherhood: policies for compatibility. Cambridge Journal of Economics 23:641-659

Dingeldey, Irene. 1999. Steuerung der Erwerbsmuster von Paaren durch nationale Steuersysteme? In $M a-$ kropolitik zwischen Nationalstaat und Europäischer Union, Hrsg. Arne Heise, 287-309. Marburg: Metropolis-Verlag.

Dingeldey, Irene. 2000. Einkommensteuersysteme und familiale Erwerbsmuster im europäischen Vergleich. In Erwerbstätigkeit und Familie in Steuer- und Sozialversicherungssystemen. Begünstigungen und Belastungen verschiedener familialer Erwerbsmuster im Ländervergleich, Hrsg. Irene Dingeldey, 11-48. Opladen: Leske + Budrich. 
Esping-Andersen, Gøsta. 1990. The three worlds of welfare capitalism. Princeton: Princeton University Press.

Eurofound. 2015. Third European Company Survey-Overview report: Workplace practices-Patterns, performance and well-being. Luxembourg: Publications Office of the European Union.

Europäische Kommission. 2012. Employment and Social Developments in Europe 2012. Luxembourg: Publications Office of the European Union.

Eurostat. 2019. Erwerbstätigenquoten nach Geschlecht, Alter und Bildungsabschluss (\%) [lfsa_ergaed]. Teilzeitbeschäftigung als Prozentsatz der gesamten Beschäftigung, nach Geschlecht und Alter (\%) (lfsa_eppga) / Durchschnittliche normalerweise geleistete Wochenarbeitsstunden in Haupttätigkeit, nach Geschlecht, Stellung im Beruf, Vollzeit-/Teilzeittätigkeit und Wirtschaftszweigen (lfsa_ewhun2). Tabellentool. https://appsso.eurostat.ec.europa.eu/nui/show.do?dataset=lfsa_ergaed\& lang=de / https://appsso.eurostat.ec.europa.eu/nui/show.do?dataset=lfsa_eppga\&lang=de / https:// appsso.eurostat.ec.europa.eu/nui/show.do?dataset=lfsa_ewhun2\&lang=de (Zugegriffen: 21. Aug. 2019).

Fernández, Esther O. 2012. Erwerbstätig oder Hausfrau? Einflussfaktoren für den Übergang erwerbstätiger Frauen in die Hausfrauentätigkeit. Drei Länder im Vergleich: Westdeutschland, Italien und Spanien. https://ediss.uni-goettingen.de/bitstream/handle/11858/00-1735-0000-0020-5F16-4/Dissertation_ Esther\%20Ochoa\%20Fern\%C3\%A1ndez.pdf?sequence=1 (Zugegriffen: 21. Aug. 2019).

Festinger, Leon, und James M. Carlsmith. 1959. Cognitive consequences of forced compliance. Journal of Abnormal and Social Psychology 58: 203-210.

Fortin, Nicole M. 2005. Gender role attitudes and the labour-market outcomes of women across OECDcountries. Oxford review of Economic Policy 21:416-438.

Frommert, Dina, Thorsten Heien und Brigitte L. Loose. 2013. Auswirkungen von Kindererziehung auf Erwerbsbiografien und Alterseinkommen von Frauen. WSI-Mitteilungen 66:338-349.

Geyer, Johannes, und Viktor Steiner. 2007. Short-run and long-term effects of childbirth on mothers' employment and working hours across institutional regimes. An empirical analysis based on the European Community Household Panel. DIW-Discussion Papers 682.

Gornick, Janet, Marcia K. Meyers und Katherin E. Ross. 1996. Supporting the employment of mothers: Policy applications across fourteen welfare states. Luxembourg Income StudyWorking Paper 139.

Grunow, Daniela, Silke Aisenbrey und Marie Evertsson. 2011. Familienpolitik, Bildung und Berufskarrieren von Müttern in Deutschland, USA und Schweden. Kölner Zeitschrift für Soziologie und Sozialpsychologie 63:395-430.

Grunow, Daniela, Katia Begall und Sandra Buchler. 2018. Gender Ideologies in Europe. A Multidimensional Framework. Journal of Marriage and Family 80:42-60.

Hans, Silke. 2006. Die Analyse gepoolter Daten mit Mehrebenenmodellen - Einstellungen zu Zuwanderern im europäischen Vergleich. Berliner Studien zur Soziologie Europas 6.

Hauser, Richard. 2008. Altersarmut in der Europäischen Union. WSI-Mitteilungen 61:125-132.

Havnes, Tarjei, und Magne Mogstad. 2009. Money for Nothing? Universal Child Care and Maternal Employment. IZA Discussion Paper 4504. http://ftp.iza.org/dp4504.pdf (Zugegriffen: 21. Aug. 2019).

Hipp, Lena. 2018. Damned if you do, damned if you don't? Experimental evidence on hiring discrimination against parents with differing lengths of family leave. SocArXiv, 27 Aug. 2018. https://doi.org/10. 31235/osf.io/qsm4x (Zugegriffen: 21. Aug. 2019).

Hipp, Lena, und Kathrin Leuze. 2015. Institutionelle Determinanten einer partnerschaftlichen Aufteilung von Erwerbsarbeit in Europa und den USA. Kölner Zeitschrift für Soziologie und Sozialpsychologie 67:659-684.

Höllinger, Franz. 1991. Frauenerwerbstätigkeit und Wandel der Geschlechtsrollen im internationalen Vergleich. Kölner Zeitschrift für Soziologie und Sozialpsychologie 43:753-771.

Huebener, Mathias, Kai-Uwe Müller, C. Katharina Spieß und Katharina Wrohlich. 2016. Zehn Jahre Elterngeld: Eine wichtige familienpolitische Maßnahme. DIW Wochenbericht 49:1159-1166.

Jaumotte, Florence. 2003. Female Labour Force Participation: Past Trends and Main Determinants in OECD Countries. OECD Economics Department Working Papers 376.

Klenner, Christina, und Sarah Lillemeier. 2015. Gender News: Große Unterschiede in den Arbeitszeiten von Frauen und Männern. Ergebnisse aus dem WSI GenderDatenPortal. WSI Report Nr. 22. http:// www.boeckler.de/pdf/p_wsi_report_22_2015.pdf (Zugegriffen: 21. Aug. 2019).

Klenner, Christina, und Tanja Schmidt. 2011. Teilzeitarbeit im Lebensverlauf von abhängig beschäftigten Frauen. In Neue Wege - Gleiche Chancen, Expertisen zum Ersten Gleichstellungsbericht der Bundesregierung, Hrsg. Ute Klammer und Markus Mutz, 253-306. Wiesbaden: VS-Verlag.

Klenner, Christina, und Tanja Schmidt. 2012. Minijobs - Eine riskante Beschäftigungsform beim normativen Übergang zum ,Adult-Worker-Modell“. WSI-Mitteilungen 1:22-31. 
Kluve, Jochen, und Sebastian Schmitz. 2014. Social Norms and Mothers' Labor Market Attachment: The Medium-Run Effects of Parental Benefits. IZA Discussion Paper 8115.

Knight, Carly R, und Mary C. Brinton. 2017. One Egalitarianism or Several? Two Decades of GenderRole-Attitude Change in Europe. American Journal of Sociology 122:1485-1532.

Koch, Angelika. 2008. Elternzeit - Teilzeit - Aus(zeit)? Teilzeitrechte in Führungspositionen. WSI-Mitteilungen 61 (11-12):612-618.

Korpi, Walter, Tommy Ferrarini und Stefan Englund. 2013. Women's Opportunities under Different Family Policy Constellations: Gender, Class, and Inequality Tradeoffs in Western Countries Re-examined. Social Politics: International Studies in Gender, State \& Society 20/1:1-40.

Kulawik, Teresa. 2005. Wohlfahrtsstaaten und Geschlechterregime im internationalen Vergleich. https:// www.fu-berlin.de/sites/gpo/systemvergleich/_konomie_und_Sozialpolitik/Wohlfahrtsstaatliche_ Konzepte_Kinderbetreuungskulturen_und_Geschlechterarrangements_in_Europa/kulawik.pdf (Zugegriffen: 21. Aug. 2019).

Kümmerling, Angelika. 2018. Geschlechtsspezifische Unterschiede in den Arbeitszeiten. Fortschritt auf der einen, Stagnation auf der anderen Seite. Duisburg: Inst. Arbeit und Qualifikation. IAQ-Report, 2018-08 http://www.iaq.uni-due.de/iaq-report/2018/report2018-08.pdf (Zugegriffen: 21. Aug. 2019).

Kümmerling, Angelika, und Patrick Lazarevic. 2016. Die Erhebungspraxis und Berechnung von Maßzahlen in der Arbeitszeitforschung: Über die Gefahr von Artefakten durch unterschiedliche Messkonzepte und Berechnungsmethoden. Zeitschrift für Arbeitswissenschaft 66:1-9.

Kümmerling, Angelika, Andreas Jansen und Steffen Lehndorff. 2008. Immer mehr Frauen sind erwerbstätig - aber mit kürzeren Wochenarbeitszeiten. IAQ-Report 2008-04:1-12 http://www.iaq.uni-due.de/ iaq-report/2008/report2008-04.pdf (Zugegriffen: 03. März 2020)

Kümmerling, Angelika, Dominik Postels und Christine Slomka. 2015. Arbeitszeiten von Männern und Frauen - alles wie gehabt? Analysen zur Erwerbsbeteiligung in Ost- und Westdeutschland. Duisburg: Inst. Arbeit und Qualifikation. IAQ-Report 2015-02 http://www.iaq.uni-due.de/iaq-report/2015/ report2015-02.pdf (Zugegriffen: 03. März 2020)

Kümmerling, Angelika, Dominik Postels und Christine Slomka. 2017. Zufriedenheit mit der Arbeitszeit - wie kann sie gelingen? Eine Analyse der Arbeitszeiten nach Geschlecht und Statusgruppen. Forschungsförderung Working Paper, Nr. 54. Düsseldorf: Hans-Böckler-Stiftung. https://www.boeckler. de/pdf/p_fofoe_WP_054_2017.pdf (Zugegriffen: 03. März 2020)

Lefebvre, Pierre, und Phillip Merrigan. 2008. Child-Care Policy and the Labor Supply of Mothers with Young Children: A Natural Experiment from Canada. Journal of Labor Economics 26:519-548.

Leibert, Tim. 2014. Frauenerwerbstätigkeit in Deutschland und Europa. Nationalatlas aktuell, 8. Leipzig: Leibniz-Institut für Länderkunde (IfL). http://aktuell.nationalatlas.de/wp-content/uploads/14_01_ Frauenerwerbstaetigkeit.pdf (Zugegriffen: 21. Aug. 2019).

Lewicki, Maria, und Berthold U. Wigger. 2013. Wer ist von Altersarmut bedroht? Wirtschaftsdienst 93:462-465.

Lewis, Jane, und Ilona Ostner. 1994. Gender and the Evolution of European Social Policies. Bremen: Zentrum für Sozialpolitik.

Lott, Yvonne. 2019. Weniger Arbeit, mehr Freizeit? Wofür Mütter und Väter flexible Arbeitsarrangements nutzen. WSI Report Nr. 47.

Macdonald, Cameron. 2009. What's culture got to do with it? Mothering ideologies as barriers to gender equity. In Gender Equality: Transforming Family Divisions of Labor, Hrsg. Janet C. Gornick und Marcia K. Meyers, 411-434. London: Verso.

Mandel, Hadas, und Moshe Semyonov. 2006. A Welfare State Paradox: State Interventions and Women's Employment Opportunities in 22 Countries. American Journal of Sociology 111:1910-1999.

Nordenmark, Mikael. 2004. Does gender ideology explain differences between countries regarding the involvement of women and of men in paid and unpaid work? International Journal of Social Welfare $13: 233-243$.

OECD. 2016. OECD Family database. LMF1.2: Maternal employment rates. http://www.oecd.org/social/ family/LMF_1_2_Maternal_Employment.pdf (Zugegriffen: 21. Aug. 2019).

Pettit, Becky, und Jennifer Hook. 2005. The Structure of Women's Employment in Comparative Perspective. Social Forces 84:779-801.

Pfau-Effinger, Birgit. 1996. Analyse internationaler Differenzen in der Erwerbsbeteiligung von Frauen. Theoretischer Rahmen und empirische Ergebnisse. Kölner Zeitschrift für Soziologie und Sozialpsychologie 48:462-492.

Pfau-Effinger, Birgit. 2000. Kultur und Frauenerwerbstätigkeit in Europa. Theorie und Empirie des internationalen Vergleichs. Leske + Budrich: Opladen. 
Pfau-Effinger, Birgit. 2012. Women's employment in the institutional and cultural context. International Journal of Sociology and Social Policy 32:530-543.

Raudenbusch, Stephen W., und Anthony S. Bryk. 2002. Hierarchical Linear Models. Thousand Oaks: Sage.

Sainsbury, Diane. 1996. Gender Equality and Welfare States. Cambridge: Cambridge University Press.

Schief, Sebastian. 2003. Arbeitszeiten in Deutschland - eine Frage der Messung? Mitteilungen aus der Arbeitsmarkt- und Berufsforschung 36:187-198.

Schulze-Buschoff, Karin, Inge Weller und Jana Rückert. 1998. Das Erwerbsverhalten von Frauen im europäischen Vergleich. Welche Faktoren beeinflussen Arbeitszeiten und Arbeitszeitwünsche? WZB Discussion Paper FS III:98-405.

Steiber, Nadia, und Barbara Haas. 2009. Ideals or compromises? The attitude-behavior relationship in mothers' employment. Socio-Economic Review 7:639-668.

Stickney, Lisa T., und Alison M. Konrad. 2007. Gender-role attitudes and earnings: A multinational study of married women and men. Sex Roles 57:801-811.

Uunk, Wilfred, Matthijs Kalmijn und Ruud Muffels. 2005. The Impact of young children on women's labour supply. A reassessment of institutional effects in Europe. Acta Sociologica 48:41-62.

Wippermann, Carsten. 2018. Frauen in Teilzeit. Lebensqualität oder Teilzeitfalle. Sozialwissenschaftliche Repräsentativbefragung im Auftrag des Bundesministeriums für Familie, Senioren, Frauen und Jugend: DELTA-Institut für Sozial- und Ökologieforschung GmbH. https://www.delta-sozialforschung. de/cms/upload/news/frauen-in-teilzeit.pdf (Zugegriffen: 25. Oktober 2019).

Yu, Wei-hsin, und Pei-lin Lee. 2013. Decomposing Gender Beliefs: Cross-National Differences in Attitudes Toward Maternal Employment and Gender Equality at Home. Social Inquiry 83:591-621.

Ziefle, Andrea, und Markus Gangl. 2014. Do Women Respond to Changes in Family Policy? A QuasiExperimental Study of the Duration of Mothers' Employment Interruptions in Germany. European Sociological Review 30:562-581.

Angelika Kümmerling 1970, Dr. phil., wissenschaftliche Mitarbeiterin am Institut Arbeit und Qualifikation der Universität Duisburg-Essen. Forschungsschwerpunkte: Arbeit und Erwerbstätigkeit in geschlechter- und internationalvergleichender Perspektive. Veröffentlichungen: Die Erhebungspraxis und Berechnung von Maßzahlen in der Arbeitszeitforschung. Über die Gefahr von Artefakten durch unterschiedliche Messkonzepte und Berechnungsmethoden. Zeitschrift für Arbeitswissenschaft, 2016 (mit P. Lazarevic); Erschöpft, unterbezahlt und ohne Lobby: Beschäftigte in der Altenpflege. In: Arbeit und Arbeitsregulierung im Finanzmarktkapitalismus: Chancen und Grenzen eines soziologischen Analysekonzepts, Hrsg. T. Haipeter, E. Latniak und S. Lehndorff. Wiesbaden 2015.

Dominik Postels 1986, ist wissenschaftlicher Mitarbeiter in den Sachgebieten Bevölkerung und Soziales im Amt für Statistik, Stadtforschung und Wahlen der Stadt Essen. Forschungsschwerpunkte: Ungleichheiten im Erwerbsverlauf, Bevölkerungsentwicklung, Quantitative Datenanalyse. Veröffentlichung: Erwerbsverläufe von Frauen und Männern mit niedrigen Versichertenrenten. Sozialpolitische Schriften, Bd. 96. Berlin 2019 (mit M. Brussig und L. Zink). 